\title{
On the nonlinear triggering of VLF emissions by power line harmonic radiation
}

\author{
D. Nunn ${ }^{1}$, J. Manninen ${ }^{2}$, T. Turunen ${ }^{3}$, V. Trakhtengerts ${ }^{4}$, and N. Erokhin ${ }^{5}$ \\ ${ }^{1}$ Department of Electronics and Computer Science, Southampton University, Southampton, UK \\ E-mail:dn@ecs.soton.ac.uk \\ ${ }^{2}$ Sodankyla Geophysical Observatory, Sodankyla, Finland \\ ${ }^{3}$ Sodankyla Geophysical Observatory, Sodankyla, Finland \\ ${ }^{4}$ Institute of Applied Physics, Nizhni Novgorod, Russia \\ ${ }^{5}$ SRI, Moscow, Russia
}

Received: 17 November 1997 / Revised: 10 June 1998 / Accepted: 16 July 1998

\begin{abstract}
VLF ground data from Porojarvi in N. Finland has been presented. Spectrograms reveal frequent occurrence of power line harmonic radiation (PLHR), originating from the Finnish power system and from heavy industrial plant. The radiation is seen to penetrate the magnetosphere since numerous occurrences of PLHR triggered emissions are seen. Risers predominate but fallers and hooks are also observed. A well-established 1D Vlasov simulation code has been used to simulate these emissions, using plausible magnetospheric data for a range of $L$ values from $L=4$ to $L=5.5$. The code is able to reproduce risers fallers and hooks in close agreement with observations. The results shed considerable insight into the generation structure of both risers and fallers.
\end{abstract}

Key words. Electromagnetics (numerical methods) · Ionosphere (wave-particle interactions) ·

Magnetospheric physics (plasma waves and instabilities).

\section{Introduction}

Power line harmonic radiation (PLHR) is a fascinating geophysical phenomenon in the near space environment that has excited much interest and lively debate during the past 20 years. PLHR lines were first recorded in the 1960s, and in the classic text by Helliwell (1965), they are visible in many of the templates, although at the time they were not recognised for what they were. It was in 1975 that Stanford University workers (Helliwell et al., 1975) positively identified PLHR lines and postulated that they originated from radiation from terrestrial power line systems. Ground VLF observations at Siple

Correspondence to: D. Nunn station Antarctica, and at the conjugate point at Roberval, Quebec in Canada revealed arrays of spectral lines in the $2-6 \mathrm{kHz}$ band. Further, analysis showed that such lines must be of magnetospheric origin. Some sets of lines have almost constant spacings of $120 \mathrm{~Hz}$, corresponding to odd harmonics of the Canadian power system. Other magnetospheric lines were found to have variable spacings that could be time varying. At the present time the general scientific consensus is that magnetospheric lines have their initiating source in terrestrial power line systems and/or heavy industrial plant. VLF harmonic radiation from power systems penetrates the ionosphere and propagates into the inner magnetosphere, where it is amplified by the unstable ambient plasma and may take the form of a selfsustaining VLF emission. Clearly, in order to understand the origin of PLHR and its magnetospheric effects it is necessary to understand the linear and nonlinear dynamics of the interaction between VLF line radiation and the ambient unstable magnetospheric plasma.

The most overt instance of nonlinear wave particle interaction in the case of PLHR radiation is the triggering of emissions by individual lines. Surprisingly in the literature few examples of PLHR TEs have been presented, and there has been little or no theoretical analysis or numerical simulation of these events. Accordingly, in this study we shall present some specific examples of magnetospheric VLF emissions triggered by PLHR. These have been observed on the ground at Porojärvi in Finland at an $L$ shell of $L=6.1$. We shall then utilise a well-established 1D Vlasov VHS code to numerically simulate the main types of triggered emission observed, in order to understand the linear and nonlinear processes by which PLHR interacts with the ambient plasma. This study has considerable environmental ramifications. There has been much debate in recent years concerning the extent to which PLHR contributes to the energy exchange between the magnetosphere and the ionosphere (Bullough, 1983, Tsurutani et al., 1981). (Emissions and chorus triggered by PLHR could be nonnegligible contributors to net precipitated particle fluxes. 


\section{Observational history of PLHR}

The literature on PLHR is impressive and vast, and here we can only give a limited review. Excellent groundbased observations of PLHR have been obtained from the magnetically conjugate pair Siple, Antarctica and Roberval, Quebec. (Helliwell et al., 1975; Park and Helliwell, 1981; Park et al., 1983). These papers report a frequent occurrence of magnetospheric lines with separations $\sim 120 \mathrm{~Hz}$. Such lines clearly originate from the Canadian power system. In the data there is clear evidence of linear and nonlinear interaction with the unstable ambient plasma. Lines are often offset from harmonics of the parent induction lines by up to $20 \mathrm{~Hz}$, and individual lines show significant spectral broadening of this order $(10-20 \mathrm{~Hz})$. Some arrays of lines with separations of $\sim 20 \mathrm{~Hz}$ are observed. These are most likely to be sidebands arising from nonlinear sideband instability (Nunn, 1986).

Ground data from the conjugate pair Halley Bay, Antarctica and St Anthony, Newfoundland showed rather different characteristics from Siple observations, presumably because the PLHR input from the ground is different. (Matthews and Yearby, 1980; Yearby et al., 1981). Indeed, Newfoundland employs a mix of 50 and $60 \mathrm{~Hz}$ power systems. Yearby and co workers found line separations to be rather variable, in the range $50-90 \mathrm{~Hz}$, and that individual lines had significant bandwidths, up to $20 \mathrm{~Hz}$. They also noted 'sideband' substructure with characteristic separations of $20 \mathrm{~Hz}$. The observed time distribution of PLHR events was quite significant, with an occurrence peak in the local afternoon, and a marked minimum on Sundays. Most events occurred at times of low or intermediate magnetic activity.

Some interesting measurements have been made in Newfoundland by Yearby et al. (1983). These authors measured the VLF wave amplitude spectra at varying distances from power lines and deduced the unbalanced currents in the lines $(\sim \mathrm{mA})$. Calculated total radiated powers were only $\sim 1$ microwatt.

In conjunction with ground data extensive satellite observations of PLHR are available. Generally observations in space tend to confirm the PLHR characteristics observed on the ground, but care in interpreting the data is needed due to the Doppler shifts and the fact that a satellite may be observing leakage from a duct some distance away. Bell et al. (1982) reported on VLF observations of PLHR on the ISEE1 satellite. PLHR was observed in only about $5 \%$ of orbits, suggesting that PLHR contributes very little to the total wave energy in the magnetosphere. Line frequencies were not always exact multiples of $60 \mathrm{~Hz}$ and line frequencies were observed to drift upwards together at rates from $+6 \rightarrow+22 \mathrm{~Hz} / \mathrm{s}$. Observations from the Ariel 3 and Ariel 4 satellites showed that PLHR was most conspicuous at quiet times when $K p<2$. The geographical distribution was concentrated over $\mathrm{N}$ America and to a lesser extent over Europe. In a recent paper of considerable interest Rodger et al. (1995) reported on PLHR observed on ISIS 1 and 2 satellites over New Zealand. Two distinct types of PLHR were observed. One of these, called "tram lines" (TLs), consisted of lines with narrow bandwidth, with zero drift, and close to harmonics of 50 or $60 \mathrm{~Hz}$. TLs clearly have their origin in power distribution systems. The other kind of PLHR were called magnetospheric lines (MLs), characterised by broader bandwidth, and no obvious correlation between line frequencies and multiples of $50 / 60 \mathrm{~Hz}$. MLs were observed normally to drift in frequency at rates up to $40 \mathrm{~Hz} / \mathrm{min}$. The drift was more often upwards though downward drifts were noted. Since ML line frequencies no longer correspond to those of the supposed terrestrial source, they would appear to be decoupled from that source and thus self-sustaining. It is not clear what role terrestrial PLHR radiation plays in either initiating MLs or in sustaining them.

\subsection{Nonlinear effects}

Clearly, since the terrestrial input signal is often fairly weak, the VLF signal injected into the magnetosphere must be amplified as a result of electron cyclotron resonance instability. The signal will also be repeatedly amplified while executing multiple reflections between hemispheres. In situations where the linear growth rate is high, PLHR may be amplified to levels $>2 \mathrm{pT}$ at which nonlinear trapping may occur in the equatorial region, and wave phenomena commonly associated with nonlinear wpi will appear.

In the literature examples of nonlinear phenomena in PLHR are common. Most observations of magnetospheric lines reveal spectral broadening (Park et al. 1983; Matthews and Yearby, 1981; Yearby et al., 1981; Park and Helliwell, 1981; Helliwell et al., 1975). Line segments with increased bandwidth and amplitude often acquire a periodicity at the two hop wave period. Also commonly noted are 'extra' sidebands with separations $\sim 20 \mathrm{~Hz}$. This frequency is of the order of the trapping frequency, which is the separation for maximum sideband growth rates.

Helliwell et al. (1975) and Park and Helliwell (1981) report capture effects in which emissions triggered by Siple pulses are either captured by a PLHR line or else suffer a discontinuous change in frequency sweep rate. Most of the observational papers above report triggering of emissions by PLHR lines. Risers are most common, but fallers and hooks also occur. None of the papers present good individual examples of triggered emissions. Helliwell et al. (1975) report bursts of emissions which merge to form something like a single chorus element, which is then repeated at the 2 hop wave bounce period.

In an inspired piece of experimental physics, the Stanford group programmed the Siple facility to simulate PLHR. Weak spectral lines with separations of 50 or $100 \mathrm{~Hz}$ were transmitted (Park and Chang, 1978; Park et al., 1983). Input wave intensities were only $\sim 0.1$ pT, but strong lines were observed at Roberval with spectral broadening and triggered emissions. The frequencies at which strong magnetospheric lines were excited did not depend upon the transmitted power, but 
rather on ambient plasma conditions. Helliwell et al. (1975) observed that chorus/periodic emissions are often 'associated' with PLHR, and that furthermore the lower starting frequency of the chorus sometimes is remarkably constant, suggesting triggering at the lower edge by a PLHR line.

The fact that PLHR is regularly observed to trigger emissions and chorus has led to the suggestion (Luette et al., 1979) that chorus is to some extent controlled by PLHR and that PLHR has a significant artificial effect on the radiation belts and upon the energy balance between the magnetosphere and ionosphere. This suggestion has however not gone unchallenged. Thorne and Tsurutani (1979, 1981), Tsurutani et al. (1979), Tsurutani and Thorne (1981) maintain that PLHR has a negligible effect on the radiation belts. We shall not join this discussion here, but a very good overall review of man-made influences on the magnetosphere will be found in Parrot and Zaslavski (1996).

\section{Theoretical background to the problem}

We shall now give some pointers to the theoretical background to the PLHR problem. The first part of a theoretical description concerns the characteristics of the radiating source, of which Kikuchi (1983) provides a very comprehensive review. The trans ionospheric injection of the weak PLHR signal into the magnetosphere, has been dealt with in Molchanov et al. (1991). To be an observable phenomenon PLHR must be substantially amplified. Observations by the Stanford group (Helliwell, 1983) give a figure of about $30 \mathrm{~dB}$ for the integrated linear growth along a field line at $L=4$ for a ducted, parallel propagating whistler. Helliwell's (1983) figure was well supported by numerical simulations of Siple triggered emissions in Nunn (1993) and Nunn et al. (1997), which required linear equatorial growth rates $>60 \mathrm{~dB} / \mathrm{s}$, a figure corresponding to integrated growth rates of about $23 \mathrm{~dB}$. If the ionospheric reflection loss does not exceed the integrated growth, then the possibility exists that PLHR induction lines could be a selfsustaining emission, but from the literature it is not clear the PLHR is always a ducted phenomenon.

When PLHR equatorial amplitudes reach levels $\sim 2 \mathrm{pT}$ (at $L=4$ ), in the case of a CW or band limited VLF wave, electron cyclotron resonance trapping occurs and the wave particle interaction process becomes nonlinear. Nonlinear trapping dynamics and the consequences for field evolution are fairly well understood, and discussed theoretically and numerically in Omura and Matsumoto (1982), Bespelov and Trakhtengerts (1986), Trakhtengerts (1995), Trakhtengerts et al. (1996) Karpman et al. (1974) and Nunn (1990). VLF emissions triggered by $\mathrm{CW}$ pulses from the Siple transmitter have been simulated in Nunn (1993), and discrete emissions observed at Halley Bay in Smith and Nunn (1998), using the same Vlasov Hybrid Simulation code that will be used in this study. The same code successfully simulated chorus observed on the Geotail satellite $(L=10)$ (Nunn et al., 1997).
In a computational and theoretical analysis (Nunn, 1986) it was shown that when nonlinear trapping takes place in a parabolically varying B field inhomogeneity, a $\mathrm{CW}$ wave is unstable to the formation of upper sidebands, the lower sidebands being damped. This means that a weak sideband wave has a growth rate greater than that of the underlying linear value. The maximum instability occurs at a sideband separation of the order of the trapping frequency, which is $\sim 20 \mathrm{~Hz}$ approx. This would certainly explain spectral broadening at nonlinear amplitudes as well as the discrete 'extra' sidebands often observed.

A major theoretical problem with PLHR is the slow upward drift of the lines at rates $\sim 20 \mathrm{~Hz} / \mathrm{min}$. If spectral broadening occurs on the upper side of the line only then this would explain a slow upward drift of frequency of radiation repeatedly crossing the equator. Another theoretical and numerical approach was expounded in Shklyar et al. (1992). The authors examined theoretically and numerically the problem of a CW pulse making a single passage of the equator at nonlinear amplitudes. It was found that a small upwards frequency shift $\sim 1 \mathrm{~Hz}$ was predicted. Repeated equatorial transits should result in slow upward drifts of the right order. Both these theoretical approaches are in some trouble when confronted by PLHR drifting downwards.

Line drift immediately entrains further theoretical headaches, since the PLHR will become effectively decoupled from its terrestrial source. Magnetospheric lines with variable or non-harmonic spacings should then constitute a self sustaining phenomenon. In this instance what then determines the set of spacings of the MLs? Nonlinear wave-wave interaction VIA nonlinear electron cyclotron resonance occurs for separations up to at least $60 \mathrm{~Hz}$ (Nunn, 1986). However the nonlinear time evolution of a series of echoing lines is a very numerically intensive problem yet to be addressed in the literature.

\section{The observations of PLHR TEs from Porojärvi}

The VLF data we shall present was obtained at Porojärvi $(69.17 \mathrm{~N}, 21.47 \mathrm{E})$ in Northern Finland during January, 1993. The antenna comprised orthogonal vertical magnetic loops. Data was sampled at $40 \mathrm{kHz}$, and processed by redundant block FFTs of length 1024 . The data is presented in the usual form of frequency/ time diagrams, with a colour coding for magnetic field spectral power, in arbitrary units. $\mathrm{F} / \mathrm{T}$ plots are available for the RH polarised component, the $\mathrm{LH}$ polarised component and for the linearly polarised component, but in this study we shall only present plots of total spectral power.

The data from Porojärvi $(L=6.1)$ is rich in PLHR events. This would appear to be partly due to the existence of strong PLHR radiation by the Finnish power system. The other factor is the apparently high ionospheric reflectivity, which is evidenced by the appearance of multiple whistler echos and other strongly echoing VLF events. The magnetospheric lines observed 
at Porojärvi must, of course, originate from the $\mathrm{N}$ Hemisphere, since the area conjugate to Porojärvi and the region to the south as far as Oulu $(65.05 \mathrm{~N}, 25.47 \mathrm{E})$ is located in the Southern Ocean between S Africa and Australia.

A full set of PLHR lines in the band $2-5 \mathrm{kHz}$ are often observed. In Finland the power system is $220 \mathrm{~V} / 3$ phase. At heavy industrial plant in Finland conversion to DC power is done using 12 pulse bridges. This results in strong harmonics being radiated at frequencies of

$f=50(c p \pm 1) \mathrm{Hz}$

where $p=12$ and $c=1,2,3,4$ etc.

Prominent pairs of lines obvious in the data are then at $1750 / 1850,2350 / 2450,2950 / 3050,3550 / 3650$ and $4150 / 4250 \mathrm{~Hz}$ etc. The lines here are 'tramlines', with constant frequencies of separation $100 \mathrm{~Hz}$ locked to the terrestrial source.

Figure 1 shows a set of strong PLHR lines, with prominent risers triggered from the PLHR doublet at $2950 / 3050 \mathrm{~Hz}$. It should be noted that PLHR are normally a weak feature, and these Finnish results show PLHR of surprising strength. It appears to be the upper line which triggers the risers. The frequency gradients are consistently in the region of $+1.0 \mathrm{kHz} / \mathrm{s}$. The first riser exhibits an initial falling frequency segment which goes down to $2800 \mathrm{~Hz}$, where it seems to 'reflect' off a weak PLHR line. The risers have a marked periodicity which is the 2 hop wave bounce time. The spectral broadening obvious in the latter emissions may be due to reflected wave energy from the previous event. Interestingly a weak narrow band faller is triggered off a line near $3.9 \mathrm{kHz}$. Polarisation analysis shows that both PLHR lines and emissions consist of a large linearly polarised component and a weak LEFT hand circularly polarised component. In accordance with Yearby and Smith (1994) this would suggest that the duct exit point is some distance from Porojärvi. Similar results for polarisation apply to all four examples in this work. With the Porojärvi data it is not possible to localise the source or duct exit point for the VLF signals observed, which would require multiple receiving sites. Direction finding from the orthogonal magnetic loops gives an ambiguous bearing for the power line at $3050 \mathrm{~Hz}$ of either north or south, and bearing for the triggered emissions of NW/SE. Now since the emissions rise to nearly $5 \mathrm{kHz}$ and the equatorial electron gyrofrequency at Porojärvi would be in the region of $4 \mathrm{kHz}$ it may be assumed that the duct exit point for the TEs is SE of Porojärvi. Furthermore for ducted VLF signals to be seen on the ground requires one to be inside the plasmapause, which also points to a southerly source.

Figure 2 shows rather different features. The strong doublet at 2950/3050 Hz triggers slow fallers with sweep rates of order $-1 \mathrm{kHz} / \mathrm{s}$. Another nonlinear phenomenon in evidence is that of 'capture'. A riser triggered by the lower hiss band is temporarily captured by the 2 lines at $2950 / 3050 \mathrm{~Hz}$. The set of lines in the band $3.5-4.5 \mathrm{kHz}$ seem to trigger a chorus-like emission consisting of a tangle of slow risers triggered off strong doublets at 3600 and $4200 \mathrm{~Hz}$. The emissions below $3.5 \mathrm{kHz}$ appear to have a bearing of ESE, while the higher frequency emissions at $4 \mathrm{kHz}$ come from a SSE direction.

Figure 3 also shows a profusion of risers triggered in the $3.5-5.5 \mathrm{kHz}$ band. These risers are very steep, particularly around $3.5 \mathrm{kHz}$, and seem to show a whistler like dispersion. It seems likely that the relevant ducts for this data example are located at a low $L$ value
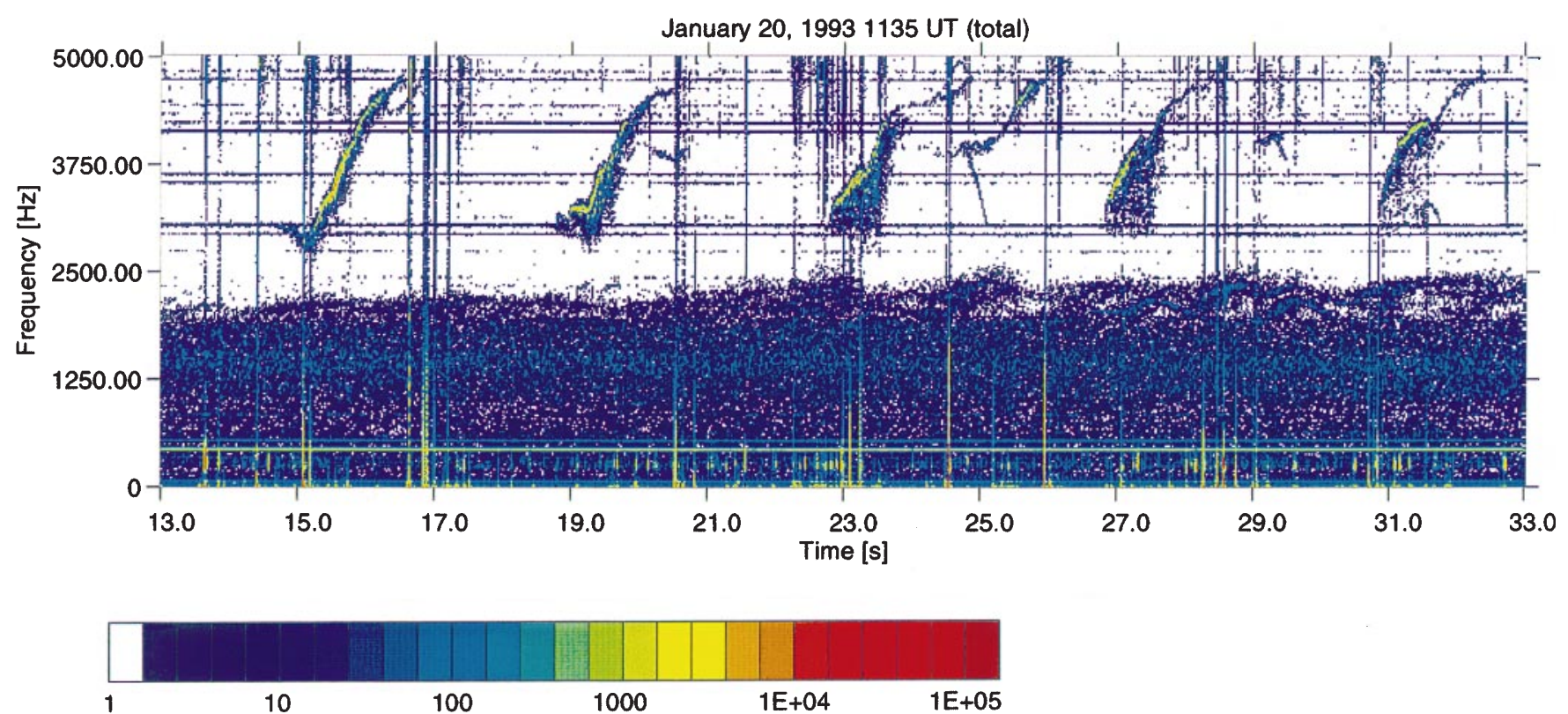

Sampling rate: $40000 \mathrm{~Hz}$ FFT-size: 1024 points Overlapping factor: 24 points Frequency indices: 257 Time indices: 800

Fig. 1. Strong rising frequency emissions triggered by a pair of prominent lines at $2950 / 3050 \mathrm{~Hz}$. The periodicity is the 2 hop wave period, and the broadening of each emission is due to the 2 hop echo of the previous one 

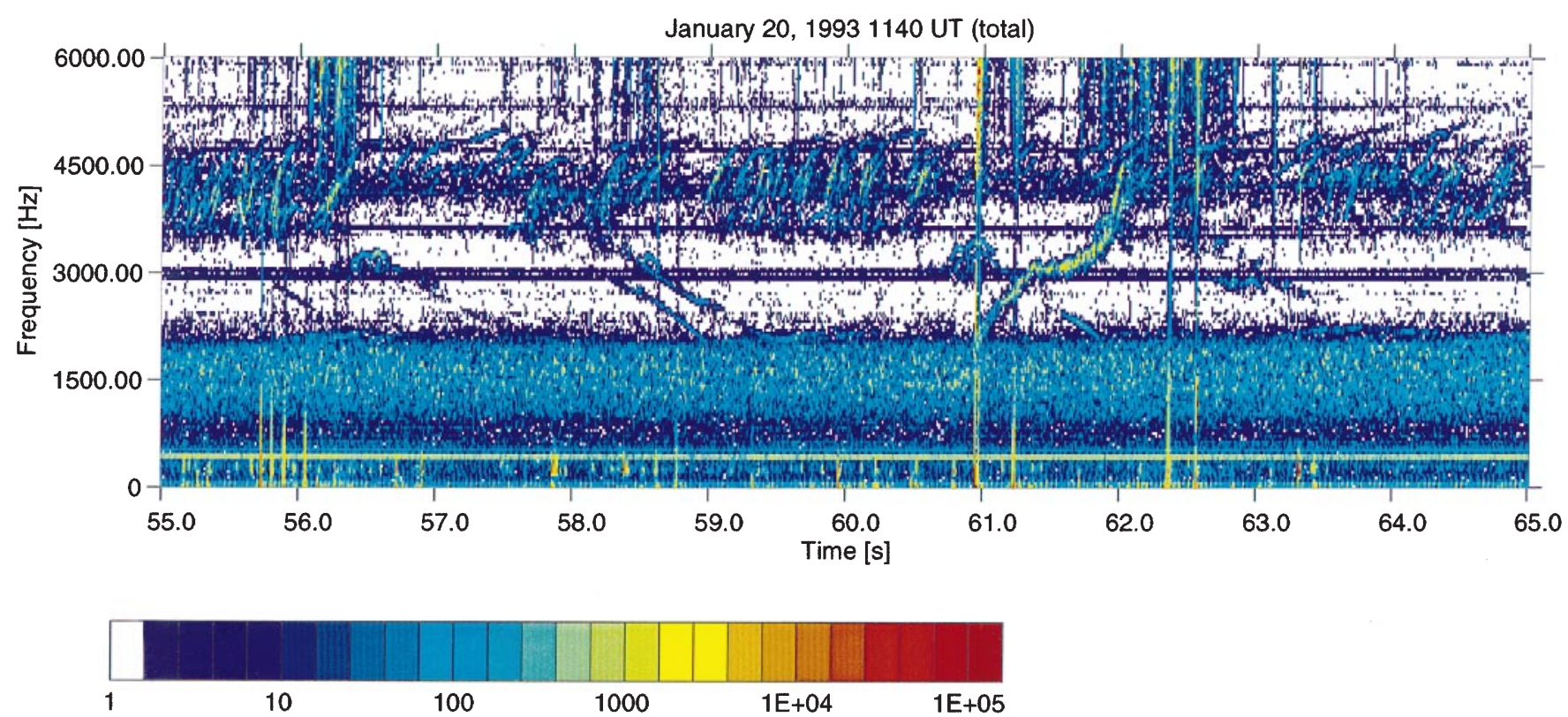

Sampling rate: $40000 \mathrm{~Hz}$ FFT-size: 512 points Overlapping factor: 12 points Frequency indices: 154 Time indices: 800

Fig. 2. Long fallers triggered by the doublet at 2950/3050 Hz. PLHR in the vicinity of $4 \mathrm{kHz}$ has triggered a disorderly chorus-like emission composed of a profusion of slow risers

$(L<4)$, which would give a whistler nose frequency above $4.5 \mathrm{kHz}$ and cause risers below this frequency to be steepened by dispersive propagation to the ground.

Small downward hook emissions are triggered off the lines at $3050 \mathrm{~Hz}$ and $3650 \mathrm{~Hz}$. An interesting feature is the lengthy powerful faller at $16 \mathrm{~s}$, which penetrates the hiss band, and turns upwards to be recaptured by the triggering line at $2950 \mathrm{~Hz}$. The hiss band is in all probability associated with another duct $/ L$ value, and will thus not degrade the nonlinear wave particle interaction process. As in Fig. 2 emissions below $3 \mathrm{kHz}$ come from a ESE direction, around $4 \mathrm{kHz}$ the bearing is more SSE, and emissions above $5 \mathrm{kHz}$ come from due south.
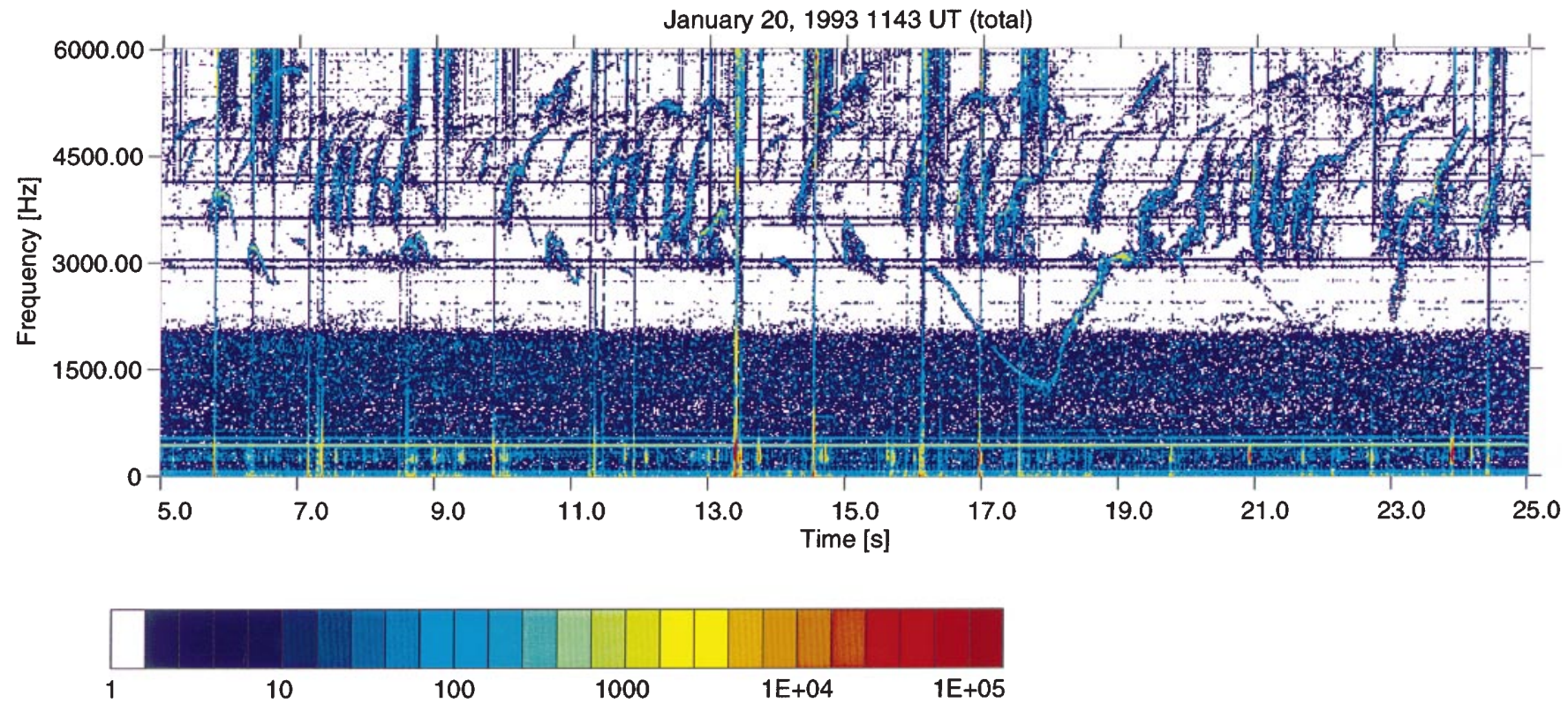

Sampling rate: $40000 \mathrm{~Hz}$ FFT-size: 1024 points Overlapping factor: 24 points Frequency indices: 308 Time indices: 800

Fig. 3. Numerous emissions triggered by pairs of PLHR at $600 \mathrm{~Hz}$ intervals. The doublet 2950/3050 Hz triggers short hooks and a pronounced slow upward hook. The $3-5-5.5 \mathrm{kHz}$ band contains numerous steep triggered risers 
Our last example in Fig. 4 shows scattered emissions clearly triggered by lines in the $4.5-6.0 \mathrm{kHz}$ band. Risers predominate, but there are some fallers and a few downward hooks. The most prominent feature is the strong slow faller which is triggered at $5350 \mathrm{~Hz}$ and descends to $4.2 \mathrm{kHz}$ in about $1 \mathrm{~s}$. All these emissions appear to come from a southerly or SE direction.

\section{The numerical simulation code}

The mathematical analysis of the VLF nonlinear wave particle interaction problem will be found in Nunn (1990). The simulation code is a 1D, Vlasov VHS electromagnetic code, which assumes a whistler wavefield that is ducted or parallel propagating to the ambient B field. This assumption is well founded since unducted VLF waves will not in general penetrate to the ground. The ambient magnetic field $B o(z)$ is assumed to vary parabolically about the equator, which effectively confines the nonlinear interaction region to the equatorial zone. This parabolic inhomogeneity effectively dictates the whole character of electron trapping and thus of the wave particle interaction process. A cold, ambient, dense electron plasma is also assumed to have a parabolic dependence on $z$, although this problem is not sensitive to the functional form of this dependence. Superimposed on this is a tenuous, hot electron population that is anisotropic and unstable to whistler waves, and thus provides the free energy for the nonlinear instability. The distribution function is modelled arbitrarily by a superposition of 6 bi Maxwellian functions
$F o(\mu, w)=\sum_{j} C_{j} \exp \left(-\mu / T_{\perp}^{j}\right) \exp \left(-(W-\mu) / T_{\|}^{j}\right)$

where $\mu$ is magnetic moment and $W$ is total energy. The set of perpendicular and parallel temperatures $T_{\perp}^{j}, T_{\|}^{j}$, and coefficients $C_{j}$ are chosen to fit observations (R. Horne, personal communication) and give an overall anisotropy of $\mathrm{A} \sim 2$ and a linear equatorial growth rate in the range $60-100 \mathrm{~dB} / \mathrm{s}$ at the equator at $3 \mathrm{kHz}$. It is easily shown (Nunn, 1990, 1993) that nonlinear growth rates for whistler waves in a parabolic inhomogeneity do not saturate with increasing amplitude, unlike in the homogeneous problem. A 1D simulation will exhibit absolute instability in the nonlinear regime, and wave amplitudes will quickly go to infinity. To achieve a successful 1D simulation it is necessary to employ a phenomenological saturation mechanism. In the time domain this takes the form of a localised damping term in which the damping rate is a rapidly increasing function of amplitude above some preset value of saturation amplitude $B_{\max }$ placed in the lower end of the nonlinear regime. Alternatively the loss process may be applied in the Fourier domain. Fortunately numerical experimentation has shown that simulation results are very insensitive to the exact form of the damping function (Nunn et al. 1977). In experimental reality the saturation process cuts in very abruptly, and is believed to arise as follows. With a nonlinear wpi process in a $3 \mathrm{D}$ ducted geometry, the 3D nonlinear resonant particle current will radiate into unducted modes, and there will be a substantial loss of wave energy out of the duct which increases sharply as amplitude increases above the threshold of nonlinearity. Another aspect of the problem is that in the nonlinear trapping regime the phase
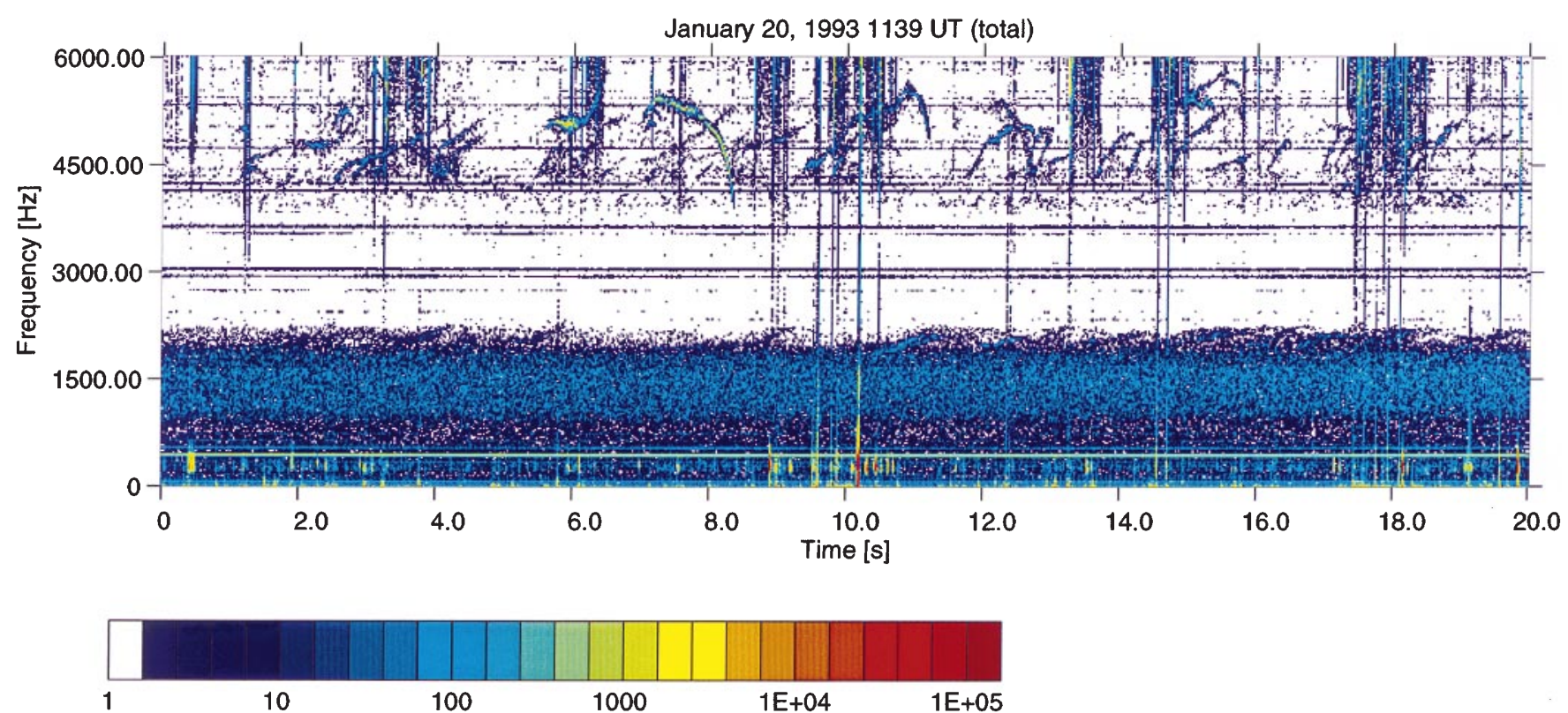

Sampling rate: $40000 \mathrm{~Hz}$ FFT-size: 1024 points Overlapping factor: 24 points Frequency indices: 308 Time indices: 800

Fig. 4. A variety of discrete PLHR triggered emissions in the band $4-6 \mathrm{kHz}$. Risers predominate, but there are a number of fallers and downward hooks 
averaged distribution function will become unstable to Langmuir waves, and the resulting Langmuir turbulence will quickly diffuse particles in the $V z$ direction thus quenching the whistler instability itself. This effect is currently under investigation. The code to be used will have a band limited formalism. A central simulation frequency $\bar{\omega}_{\mathrm{o}}$ is defined, and at each time step the wave field is bandpass filtered by fast Fourier transform and inverse fast Fourier transform (FFT/IFFT) to the band $\bar{\omega}_{\mathrm{o}} \pm \delta \bar{\omega}_{\mathrm{BW}}$, where $\delta \bar{\omega}_{\mathrm{BW}}$ is the simulation half bandwidth, typically of order $20 \mathrm{~Hz}$. The centre frequency $\bar{\omega}_{\mathrm{o}}$ is freely variable and is continuously adjusted during the simulation. For the triggered emission problem a further complication is necessary. It was shown in Nunn et al. (1977) that spatial gradients of wave number/frequency are very significant in the triggered emission problem, and constitute a significant part of the mechanism responsible for the observed sweeping frequency. In the recent version of the code, at each time step the wave number as a function of $z, k(z)$ is determined, and an $m$ th order least mean squares (LMS) polynomial fit $k_{m}(z)$ is found. The wave field is then spatially bandpass filtered to $k_{m}(z) \pm \delta \bar{\omega}_{\mathrm{BW}} / V g$, thus allowing the wave field to establish marked spatial gradients of frequency and wave number. In the simulations in this study we shall employ $m=1$, i.e. a linear fit, which was found to give the best performance.

The VHS method used here sets up a 4D phase space simulation box and grid in the variables $z, V z, \psi$, and $V_{\perp}$. The $z$ grid is fixed and contains the equatorial trapping region for the case $B=B_{\max }$. (plus $20 \%$ ). The number of spatial grid points $N_{z}=1024$ must be enough to resolve the simulation bandwidth plus the spatial variation of $k$ across the box. The grid spacing must be small enough to ensure satisfactory integration of the simulation particle equations of motion. The variable $\psi$ is gyrophase, and $N_{\psi}=20$. The centre of the $V z$ range resolved is matched to the local resonance velocity $V_{\text {res }}$. The velocity width resolved, $\Delta V_{z}$. is given by

$\Delta V_{z}=2 \delta \bar{\omega}_{\text {BW }} \mathrm{d} V$ res $/ \mathrm{d} \bar{\omega}+4 V_{\text {tr }}$

where $V_{\mathrm{tr}}$ is the resonant particle trap width corresponding to $B=B_{\max }$. A grid of $N_{v}=40$ was found to adequately resolve significant structures in velocity space, which are of course the resonant particle traps. The remaining coordinate is $V_{\perp}$, which in this problem is 'weak'. Only a narrow pitch angle range from 45-65 degrees is a significant contributor to nonlinear wpi, and very few grid points $N v_{\perp}$ are needed. The simulations in this work will employ $N v_{\perp}=3$ or even $N v_{\perp}=1$. In the context of Vlasov simulations this is perfectly legitimate, something which may not be said of PIC codes.

The numerically intensive part of the code lies in the computation of nonlinear resonant particle current $J_{\text {res }}(z, t)$. This is accomplished using the Vlasov Hybrid Simulation (VHS) method which is highly efficient and well studied to the problem at hand. The method is described in detail in Nunn (1993). The 4D phase space box is evenly filled with simulation particles with a density of at least 1.2 per grid cell. The program continuously follows these particles, which represent phase trajectories. When a particle leaves the phase box it is discarded, since in this problem it is non-resonant with the ambient wavefield. Where phase fluid flows into the box new particles are inserted into the phase fluid at the phase box boundary. This has to be done with some care. Using Liouville's theorem, distribution function $F$ is conserved along these phase trajectories and is thus known, though in this configuration $\Delta W$ or integrated energy change is evaluated along each trajectory. The key operation in VHS codes is the INTERPOLATION of $F($ or $\Delta W$ ) from the particles onto the phase space grid. This is achieved using a variant of the area weighting scheme employed in PIC codes. (Nunn, 1993). Once $\Delta W$ is defined on the phase space grid it is a simple matter to compute the resonant particle current by integration over velocity space.

VHS differs from other Vlasov codes (Candy 1996; Klimas et al., (1994)) by its use of interpolation. This confers immediate and obvious benefits. Because of it there is no need to assign some small phase volume to each simulation particle. Simulation particles may be inserted into the phase fluid at will (or removed), and there is no problem in dealing with a flux of phase fluid into the simulation box. Since by Liouville's theorem density is conserved there is no tendency for particles to bunch and leave grid points uncovered. It is only required that particle density be above some minimum level sufficient to ensure that all grid points have at least one particle in an adjacent cell. Another consequence of interpolation is that it confers robustness to filamentation in the distribution function. Distribution fine structure will undersampled by our assembly of particles, and the resulting resonant particle current will be corrupted by a low level of aliased broadband noise. In the unlikely event that this is a problem, one need only increase particle density.

In conclusion VHS has to be seen as a highly efficient, relatively simple and robust method for numerical simulation of hot plasma, where resolution of $F$ in velocity space is required. It is far superior to PIC codes, a property shared by all simulation techniques that essentially push $\delta F$.

\section{The simulation results}

We will show four simulation runs, one for each of the main types of triggered emission observed, namely a riser, a faller, an upward hook and a downward hook. The duct location and corresponding plasma parameters at the equator are, of course, not known for individually observed TE events. We shall select plausible parameters in each case. What we will not do is select each simulation plasma parameter in turn and see how simulation results depend upon it. This would be a very useful exercise but currently too expensive for our available computer resources. All the input and simulation parameters used in these four runs are presented in Table 1.

Case A. Riser The first simulation will be of a long stable riser triggered by a single line at $3650 \mathrm{~Hz}$. The 
doublet separation of $100 \mathrm{~Hz}$ exceeds the normal bandwidth of the code $(20-60 \mathrm{~Hz})$ and in any case nonlinear wave-wave interaction via cyclotron resonant interaction is very weak at a separation of $100 \mathrm{~Hz}$, which is five times the resonant trapping frequency separation of $20 \mathrm{~Hz}$. The lower line in the doublet would only have a significant effect if the riser were triggered from the lower line. The simulation input signal at $z=z_{1}$ is a weak key-down CW signal with an amplitude of $0.1 \mathrm{pT}$. We assume that the duct is inside the plasmapause and well to the southeast of Porojärvi. The chosen $L$ value is 4.4 , in the vicinity of Oulu. The linear growth rate at the equator is taken to be $79 \mathrm{~dB} / \mathrm{s}$ at $3650 \mathrm{~Hz}$. The code is driven by a three $V_{\perp}$ 'beams', with pitch angles of 54, 61 and 66 degrees. The hot plasma distribution function is modelled as 6 bi Maxwellians, with an anisotropy of 2.5 . The saturation amplitude employed in the code is $B_{\max }=6.9 \mathrm{pT}$, which permits highly nonlinear resonant particle trapping. The total bandwidth of the simulation matched filter is set at
$37 \mathrm{~Hz}$, which is of the order of the maximum trapping frequency. The global bandpass filter has a width of $1100 \mathrm{~Hz}$.

The field data exiting from the right hand end of the simulation box is fed into a buffer, where it is processed by overlapping block FFTs, which are Hamming shaded and have a frequency resolution of $3 \mathrm{~Hz}$. Figure 5 shows a histogram equalised, shaded IDL contour plot of spectral power as a function of time. The frequency rises from $3.6 \mathrm{kHz}$ to $5.5 \mathrm{kHz}$ in $2 \mathrm{~s}$, and reaches a sweep rate of $1600 \mathrm{~Hz} / \mathrm{s}$, which is a typical observed value. It should be realised that emissions observed on the ground will have the extra dispersion due to propagation from the equator to Earth, but since the nose frequency is probably in the region $f \sim 3.6 \mathrm{kHz}$, it is unlikely that risers will be steepened by this means. The most common emission to be triggered is the riser and this simulated one bears a close resemblance to those observed, particularly the examples in Figs. 1 and 3.

Table 1. Input data used for numerical simulations

\begin{tabular}{lllll}
\hline & $\begin{array}{l}\text { Run A: } \\
\text { riser }\end{array}$ & $\begin{array}{l}\text { Run B: } \\
\text { faller }\end{array}$ & $\begin{array}{l}\text { Run C: } \\
\text { hook/down }\end{array}$ & $\begin{array}{l}\text { Run D: } \\
\text { hook/up }\end{array}$ \\
\hline Bin $(\mathrm{pT})$ & 0.1 & 0.1 & 0.1 & 0.1 \\
$L$ Shell & 4.4 & 4.1 & 4.1 & 4.1 \\
$\omega_{\mathrm{e}}(\mathrm{Hz})$ & $11 \mathrm{~K}$ & $12.45 \mathrm{~K}$ & $9 \mathrm{~K}$ & $9 \mathrm{~K}$ \\
$\mathrm{Ne}\left(\mathrm{cm}^{-3}\right)$ & 400 & 400 & 400 & 400 \\
$\gamma(\mathrm{dB} / \mathrm{s})$ & 79 & 99 & 70 & 90 \\
$B \max (\mathrm{pT})$ & 6.9 & 6.9 & 4.9 & 5.9 \\
$F(\mathrm{~Hz})$ & 3650 & 4150 & 2950 & 2950 \\
$N v \perp$ & 3 & 3 & 1 & 1 \\
$\alpha(\mathrm{degs})$ & $54,61,66$ & $51,62,67$ & 61 & 61 \\
$\omega_{\text {BW }}$ global $(\mathrm{Hz})$ & 1100 & 878 & 626 & 686 \\
$\omega_{\text {BW matched }(\mathrm{Hz})}$ & 37 & 29 & 21 & 23 \\
Grid $N v, N_{\psi}, N z$ & $40,20,1024$ & $40,20,1024$ & $40,20,512$ & $40,20,1024$ \\
\hline
\end{tabular}

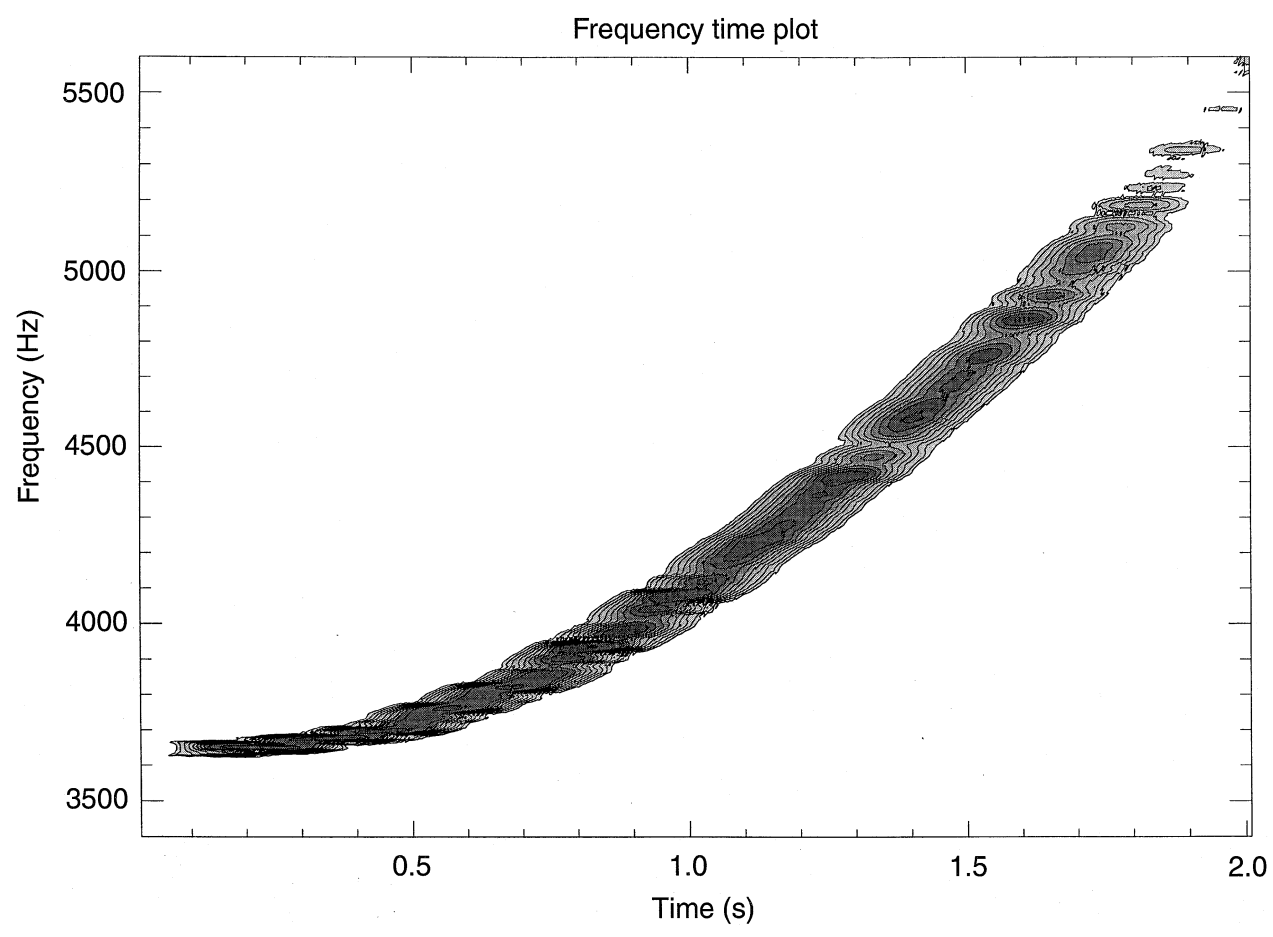

Fig. 5. Case A. Riser. Frequency time plot of the exit wavefield, shown as a shaded IDL contour plot. A key-down weak $\mathrm{CW}$ input signal of $0.1 \mathrm{pT}$ gives a long stable rising emission with a sweep rate of $2 \mathrm{kHz} / \mathrm{s}$ 
The process by which a VLF emission is triggered is as follows. The input signal introduced at $z=z_{1}$ is very weak, but undergoes linear amplification in propagating to the equatorial region, indeed the integrated linear amplification across the simulation box for these simulations if of the order of $25 \mathrm{~dB}$. If the wave amplitude in the equatorial zone reaches the level at which nonlinear trapping commences (about $1 \mathrm{pT}$ ), then a self sustaining VLF generation region (GR) may be set up. Very weak PLHR lines which do not reach the nonlinear threshold at the equator do not trigger, something that may be seen in the data.

Figure 6 shows graphs of wave profile (in pT), and nonlinear currents $J_{r}$ and $J_{i}$ as functions of $z$ at $t=1.442 \mathrm{~s}$. The current $J_{r}$ is the component in phase with the wave electric field (heavy line), and $J_{i}$ is the component in phase with the wave $B$ field. These currents are normalised such that a $\mathrm{CW}$ wave at $z=0, f=3.6 \mathrm{kHz}$ at the saturation amplitude $B_{\max }$ $(=6.9 \mathrm{pT})$ would give rise to a linear resonant particle current $J_{\mathrm{r}}=-1$. Apart from sideband oscillations, which manifest themselves as oscillations in wave amplitude profile at the trapping length, these currents exhibit the functional form to be expected as a result of trapping in a parabolic inhomogeneity. (Nunn, 1990). Note, in particular, the pronounced negative peak in $J_{i}$ which is the primary cause of the sweeping frequency in an emission. This figure represents a snapshot of a typical GR for a rising frequency emission. The GR resembles a 'VLF SOLITON'. It is a self consistent, nonlinear structure that is self-sustaining and has a zero field input (Nunn et al., 1997). The structure is surprisingly dynamically stable. The profile remains roughly constant in time, though time dependent sideband activity will occur in practice. However in the case of hooks the GR may change its structure to a different configuration. It is clear that in order to maintain a wave profile at a constant position on the field line a minimum linear equatorial growth rate is required. In the present simulation that figure is about $52 \mathrm{~dB} / \mathrm{s}$. Below this level there is no triggering and the plasma is absolutely stable, but convectively unstable. Above this figure the plasma exhibits absolute and convective instability but ONLY in the nonlinear regime. Almost any input signal (of sufficient size) will then result in the setup of a GR and trigger an emission. This confirms a very important aspect of these simulations, that is the triggering is a very repeatable and stable phenomenon. Following the initial stage of linear amplification the nonlinear regime is entered, which results in the set up of a stable nonlinear soliton like structure. Interestingly almost identical behaviour was found in numerical studies of electrostatic solitary waves in Omura et al. (1996).

Nonlinear growth rates increase rapidly with amplitude $\left(\propto B^{3 / 2}\right)$ above the trapping threshold. Inspection of the lower panel of Fig. 6 shows nonlinear growth rates about 5 times the linear value. To achieve dynamical stability the GR must shed a substantial fraction of this power input via the mechanism of nonlinear unducting loss. For a strong emission about $80 \%$ of input power will be lost in this way.
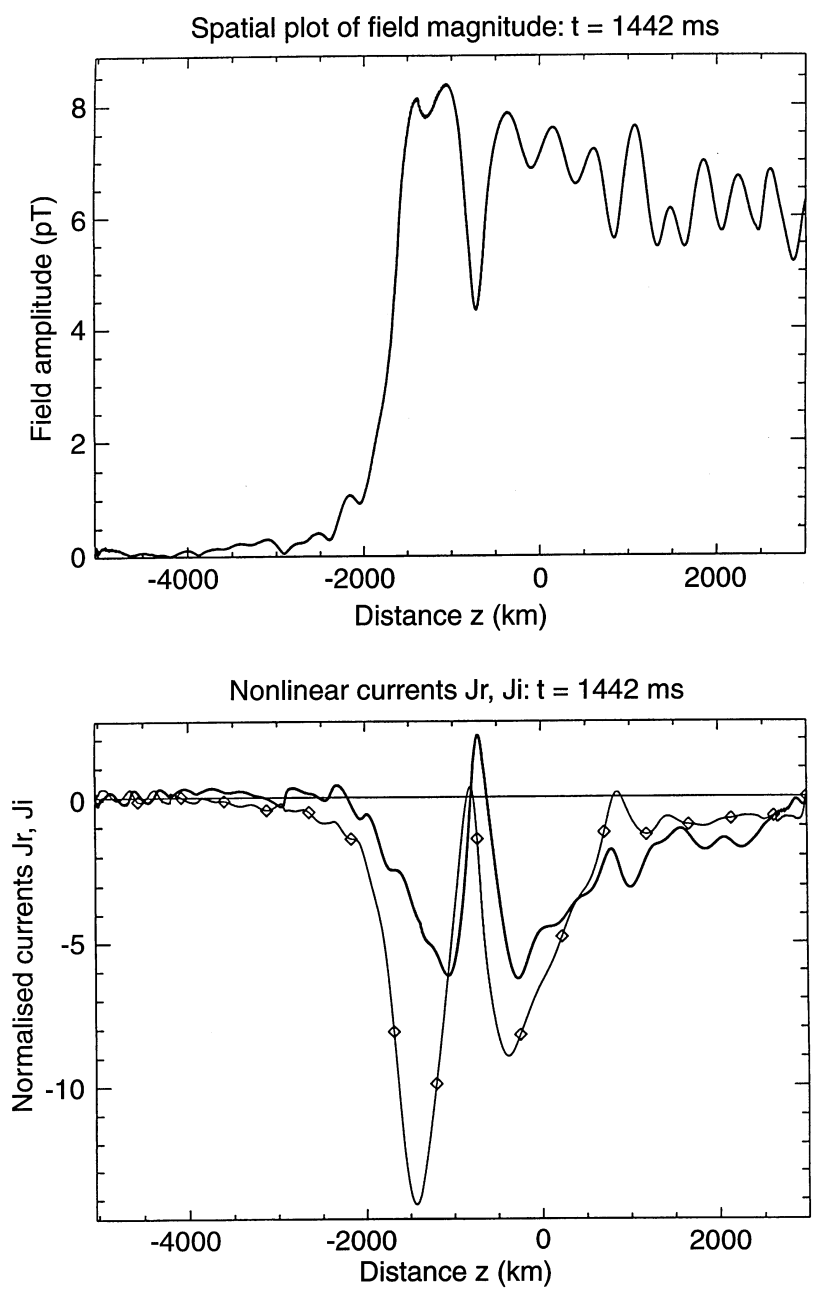

Fig. 6. Case A. Plots of wave field amplitude (pT), in phase nonlinear current $\left(J_{r}\right)$ and out of phase current $\left(J_{i}\right)$ as functions of $z$ at $t=1.442 \mathrm{~s}$. The heavy line is $J_{r}$, the light line $J_{i}$. This represents a snapshot of the structure of a typical VLF generation region for a rising frequency emission

The emission GR is normally characterised by a sweeping frequency. The origin of this can be seen by inspecting a derivative of the dimensionless field equation, Nunn(1990).

$\frac{\partial \omega}{\partial t}=\frac{\partial^{2} \phi}{\partial t^{2}}=V g^{2} \frac{\partial^{2} \phi}{\partial t^{2}}+\frac{V g^{2} \omega_{\mathrm{o}}}{k_{\mathrm{o}}} \frac{\partial}{\partial z}\left(\frac{J_{i}}{R}\right)-\frac{\omega_{\mathrm{o}} V g}{k_{\mathrm{o}}} \frac{\partial}{\partial t}\left(\frac{J_{i}}{R}\right)$

Here $\phi$ is the additional phase over 'base' phase, $V g$ is group velocity and $R$ is dimensionless wave amplitude. The last term clearly cannot change frequency by more than a few Hertz, as may be seen by integrating wrt $t$ for each $z$. The second term may make a significant contribution to sweep rate, and would normally average to $\sim<300 \mathrm{~Hz} / \mathrm{s}$ in a riser GR. It has become apparent that the first term, which is purely advective, is the major contributor to sweep rate. This version of the VHS code allows a linear variation of average wave number to develop across the simulation box. Figure 1 plots the spatial wave number gradient $(-\mathrm{d} k / \mathrm{d} z) V g^{2}(\mathrm{~Hz} / \mathrm{s})$ as a function of time. The gradient increases steadily to reach a value of $1400 \mathrm{~Hz} / \mathrm{s}$. The other term contributing to 
sweep rate is $\Phi=V g^{2} \frac{\omega_{0}}{k_{0}} \frac{\partial}{\partial z}\left(\frac{J_{i}}{R}\right)$. Figure 9 plots this quantity as a function of $z$ at $t=2 \mathrm{~s}$, though it is rather noisy due to sideband activity. It will be seen that a significant positive peak $\sim+800 \mathrm{~Hz} / \mathrm{s}$ is reached on the left hand side of the GR. We thus see that $\Phi$ is a secondary direct contributor to $\mathrm{d} f / \mathrm{d} t$, and also that the spatial gradient of $\Phi$ is responsible for the setting up of the spatial gradients of $k$.

With a bandwidth of $37 \mathrm{~Hz}$ this simulation is highly nonlinear and characterised by the trapping of resonant particles for long times. The VHS code readily provides distribution function plots as a diagnostic. Figure 8 plots the related quantity $\Delta W$, integrated energy change, as a function of gyrophase and $V^{*}$. Note that the phase in the diagram is relative to base phase and not relative to local $\mathrm{E}$ field. This is the case for all these wire diagrams. The result is presented as an IDL wire diagram, at $z=0$. The block of particles with large values of $\Delta W$ are stably trapped particles. Other particles with small $\Delta W$ are 'passing' particles. This graph confirms the fact that when there is strong trapping in inhomogeneous media, then stably trapped particles control the dynamics of the nonlinear wave particle interaction process.

Case B. Faller. The second simulation will be of a faller triggered by a higher frequency PLHR line at $4150 \mathrm{~Hz}$, which is the lower of the doublet 4150 / $4250 \mathrm{~Hz}$. Simulation parameters will be similar to run A except that linear growth rate is higher at $99 \mathrm{~dB} / \mathrm{s}$. The simulation bandwidth of the matched filter is set narrower at $29 \mathrm{~Hz}$, since in reality fallers are observed to have a narrower bandwidth. Figure 10 shows the $f / t$ diagram of the exit wave field. The sweep rate increases steadily to a value $\sim-1000 \mathrm{~Hz} / \mathrm{s}-\mathrm{a}$ typical value. The emission terminates at $2900 \mathrm{~Hz}$. At this point the linear growth rate has fallen to $\sim 45 \mathrm{~dB} / \mathrm{s}$, which is below the absolute instability level.

Figure 11 represents a snapshot of the falling tone GR at $t=967 \mathrm{~ms}$. Partly as a consequence of the high

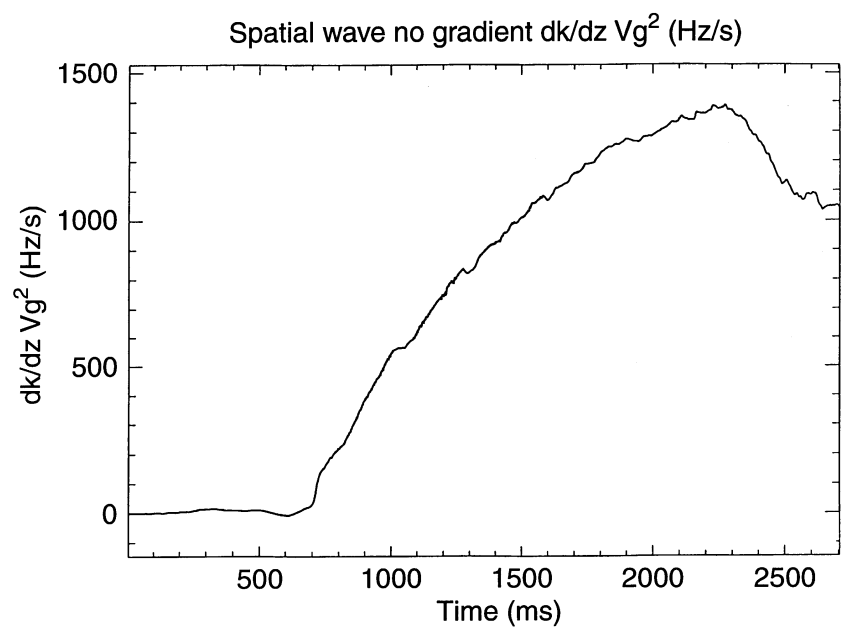

Fig. 7. Case A. Plot of spatially averaged wave number gradient across the simulation box, as a function of time. The quantity plotted is $\left(-\mathrm{d} k / \mathrm{d} z \cdot V g^{2}\right)(\mathrm{Hz} / \mathrm{s})$. The final value of $+1400 \mathrm{~Hz} / \mathrm{s}$ indicates that spatial wave number gradients are a dominant cause of frequency sweep in VLF emissions

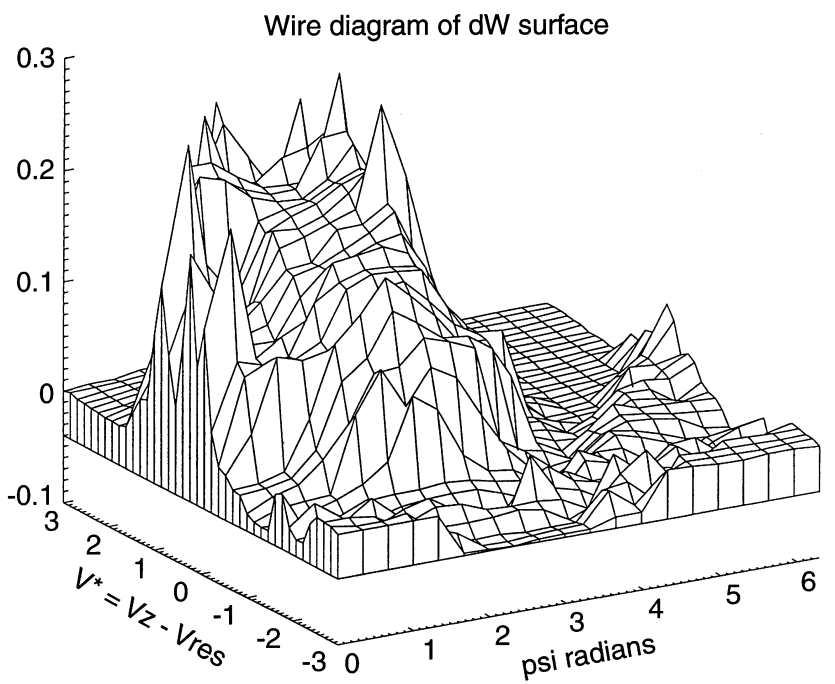

Fig. 8. Case A. Plot of integrated energy change $\Delta W$ in $\psi / V^{*}$ space at $z=0 . \Delta W$ is in dimensionless units. The plot is presented as an IDL wire diagram and shows the dominance of stably trapped particles

Plot of phi $=A d / d z(J i / R)(H z / s)$ and IRI $(p T)$

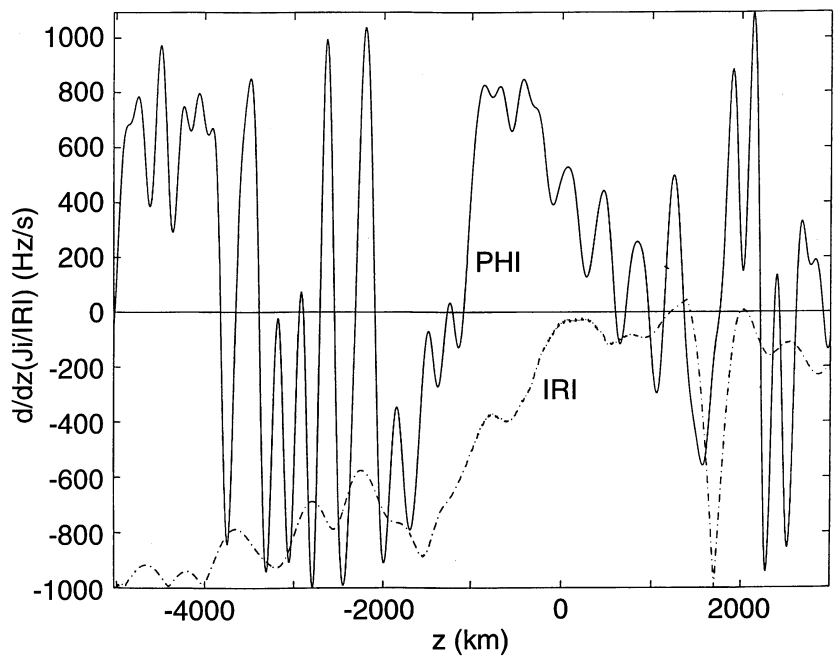

Fig. 9. Case A. Plot of $\left\{V g^{2} \omega_{\mathrm{o}} / k_{\mathrm{o}} \cdot \mathrm{d} / \mathrm{d} z\left(J_{i} / R\right)\right\}$ in $\mathrm{Hz} / \mathrm{s}$ as a function of $z$ at $t=2 \mathrm{~s}$. A prominent peak $\sim+800 \mathrm{~Hz} / \mathrm{s}$ will be noticed at the left hand end of the GR amplitude profile

growth rates and high nonlinearity, the wave profile extends well upstream of the equator, and enables trapping to take place there. Simple trapping theory predicts a positive value of $J_{i}$ in this region, giving a negative value for $\frac{\partial}{\partial z}\left(\frac{J_{i}}{R}\right)$. This causes negative wave number gradients to be set up, giving a falling frequency. Figure 12 shows the spatial gradient of $k$ as a function of time, and builds up steadily to a maximum value of $-1200 \mathrm{~Hz} / \mathrm{s}$. We thus see two distinct GR structures, one for a faller and one for a riser. Hooked emissions occur when the structure changes type between these two. For example the slow upward hook occurs because the power input falls with falling frequency, the profile slips downstream, and a riser 


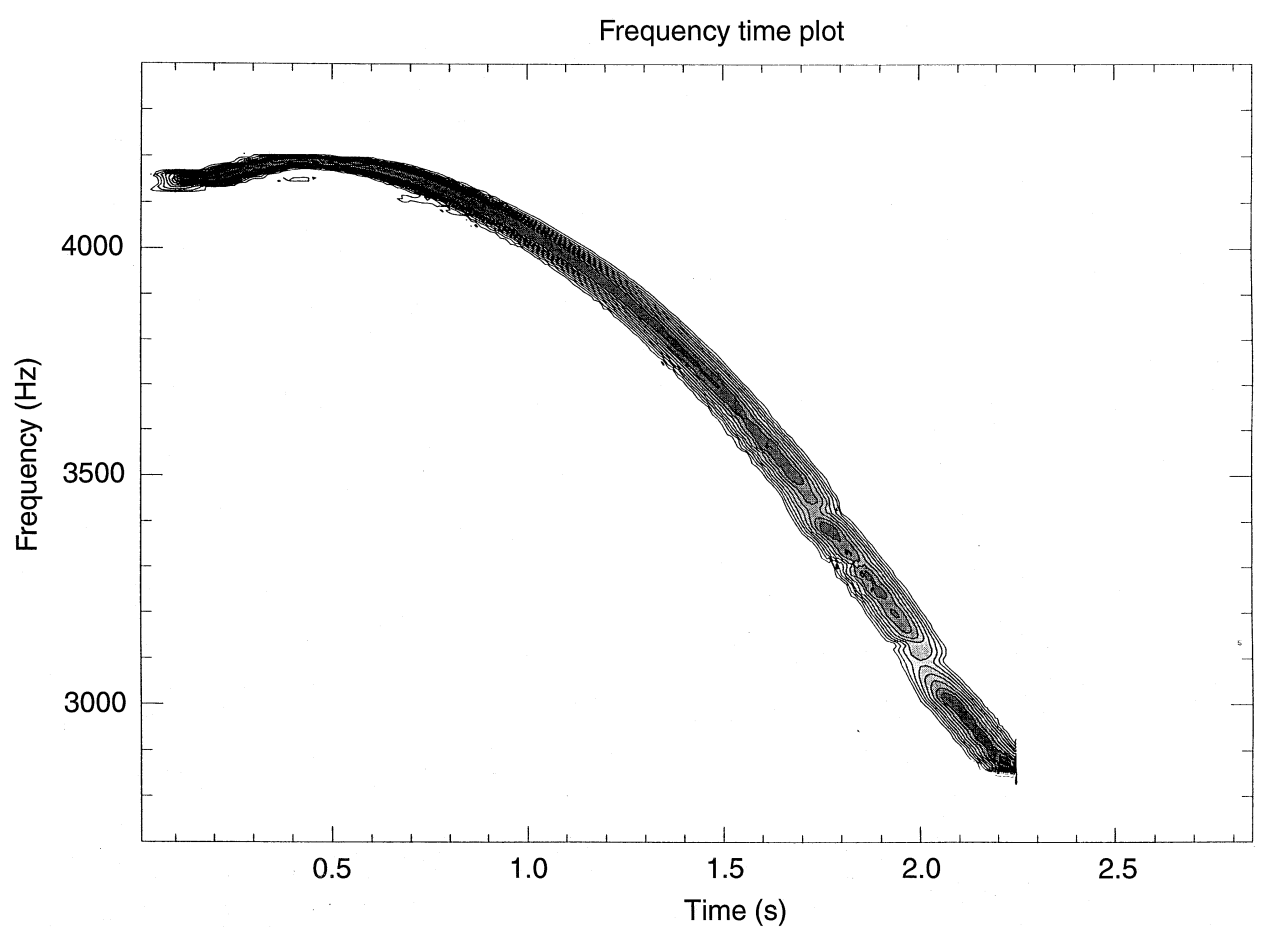

Fig. 10. Case B. Steep faller. Frequency time diagram of the exit wave field. The sweep rate is about $-1 \mathrm{kHz} / \mathrm{s}$. The emission terminates at $t=2.2 \mathrm{~s}$ due to the decreasing linear growth rates at lower frequencies
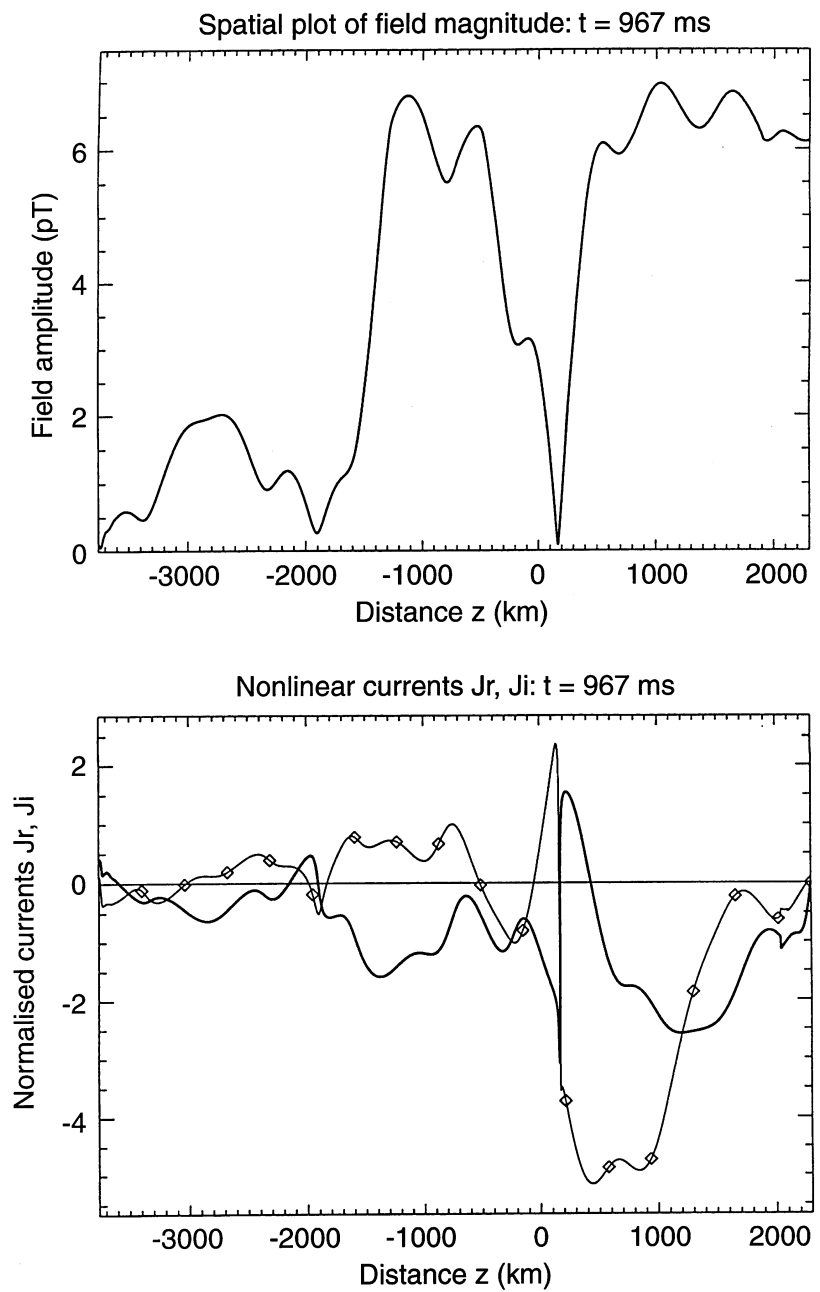

Fig. 11. Case B. Snapshot of the faller generation region at $t=967 \mathrm{~ms}$. The amplitude profile extends well upstream of the equator. Particle trapping in this positive inhomogeneity region results in $J_{i}>0$, which gives rise to the falling tone

GR results. With these PLHR simulations both upward and downward hooks were common-one half of all simulated fallers ended in an upward hook, the others terminated.

Figure 13 is a wire diagram of $\Delta W$ at $t=1.5 \mathrm{~s}$ and $z=-1000 \mathrm{kms}$. At this point resonant particle are trapped in a positive inhomogeneity region and have negative $\Delta W$ as expected.

Case C. Downward hook. The third simulation will be of a downward hook triggered off a lower PLHR line at $2950 \mathrm{~Hz}$. Again an $L$ value of 4.1 well to the SE of Porojärvi is assumed. This simulation has a lower saturation amplitude of $4.9 \mathrm{pT}$ and is thus less nonlinear. The code is driven by a single beam with a pitch angle of $61^{\circ}$. Figure 14 shows the $f / t$ diagram for this case. At $t=3 \mathrm{~s}$ the linear growth rate reaches $175 \mathrm{~dB} / \mathrm{s}$, a direct consequence of the assumed bi-Maxwellian distribution. With such a high power input the wave amplitude profile spreads upstream. Particle trapping then occurs for $z<0$ giving $J_{i}>0$, which converts the GR to the faller type. The form of this downward hook agrees well with those observed, particularly in Fig. 4.

Figure 15 represents a snapshot of the riser GR at $t=1349 \mathrm{~ms}$. The structure strongly resembles that of run A. Later in the simulation Fig. 16 shows a snap of a faller GR at $t=4047 \mathrm{~ms}$, which closely resembles that in run $\mathrm{B}$.

Figure 17 plots spatial gradient of $k$ as a function of time which reaches a positive peak of $+500 \mathrm{~Hz} / \mathrm{s}$, but then falls to $-500 \mathrm{~Hz} / \mathrm{s}$ after the hook discontinuity. Figure 18 presents a $\Delta W$ wire diagram at $t=3 \mathrm{~s}$ and at $z=+2000 \mathrm{kms}$. The trapping topology is more complex in this case.

Run D. Upward hook. The last run will be of an upward hook triggered from a PLHR line at $2950 \mathrm{~Hz}$. Param- 


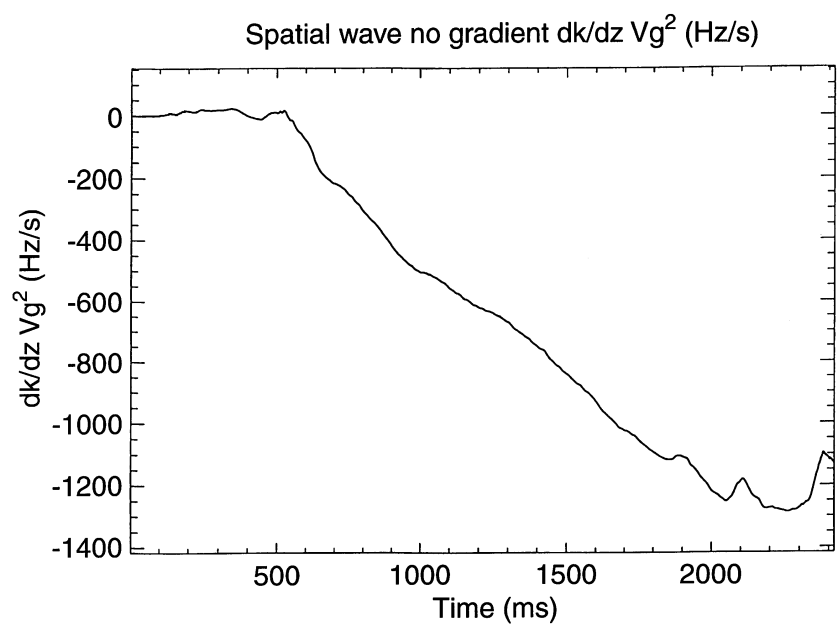

Fig. 12. Case B. Plot of spatial wave number gradient $\left(-\mathrm{d} k / \mathrm{d} z . V g^{2}\right)$ in $\mathrm{Hz} / \mathrm{s}$ as a function of time. Its value steadily decreases to $-1200 \mathrm{~Hz} / \mathrm{s}$

eters for this run will be found in Table 1. Figure 19 plots the $f / t$ diagram for this case. At $t=2.5 \mathrm{~s}$ the linear growth rate falls to about $50 \mathrm{~dB} / \mathrm{s}$, close to the absolute instability limit. At this point the wave amplitude profile slips downstream and particle trapping is then confined to the negative inhomogeneity region for $z>0$. The current $J_{i}$ then becomes negative and the GR converts to the riser type. At this point the GR could well be 'lost' and the emission terminate. It is not clear what determines whether the emission terminates or whether an upward hook is produced. Figure 20 shows a snapshot of a faller GR at $t=1658 \mathrm{~ms}$ and Fig. 21 reveals a GR of the riser type at $t=4146 \mathrm{~ms}$. The switchover between GR types is apparent in Fig. 22, where the spatial gradient flips over from $-500 \mathrm{~Hz} / \mathrm{s}$ to $+800 \mathrm{~Hz} / \mathrm{s}$. Finally Fig. 23 shows a $\Delta W$ wire diagram in
Wire diagram of $\mathrm{dW}$ surface

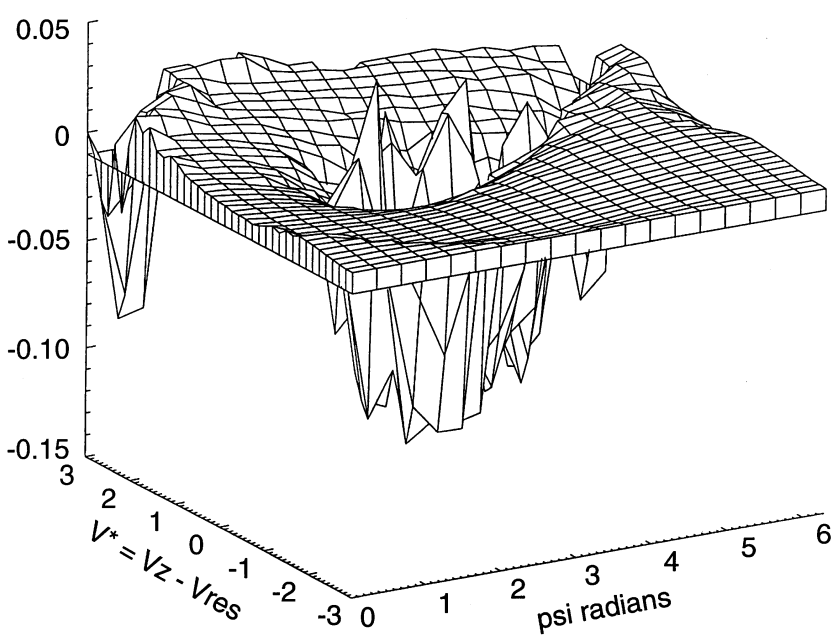

Fig. 13. Case B. Wire diagram representation of $\Delta W$ in the $V^{*} / \psi$ plane for the faller, at $t=1.5 \mathrm{~s}$ and at $z=-1000 \mathrm{~km}$. The trapped particles have $\Delta W<0$ as one would expect from trapping in a positive inhomogeneity

the faller segment at $t=1.5 \mathrm{~s}$ and at $z=-1000 \mathrm{kms}$. The distinctive bunch of trapped particles with $\Delta W<0$ is apparent.

\section{Conclusions}

We have presented ground observations from Porojärvi, Finland, of VLF emissions triggered by PLHR induction lines. Previous papers have, of course, reported such emissions, but here we have studied them in some detail, explained their plasma physical origin and simulated a variety of emission forms observed. Prom-

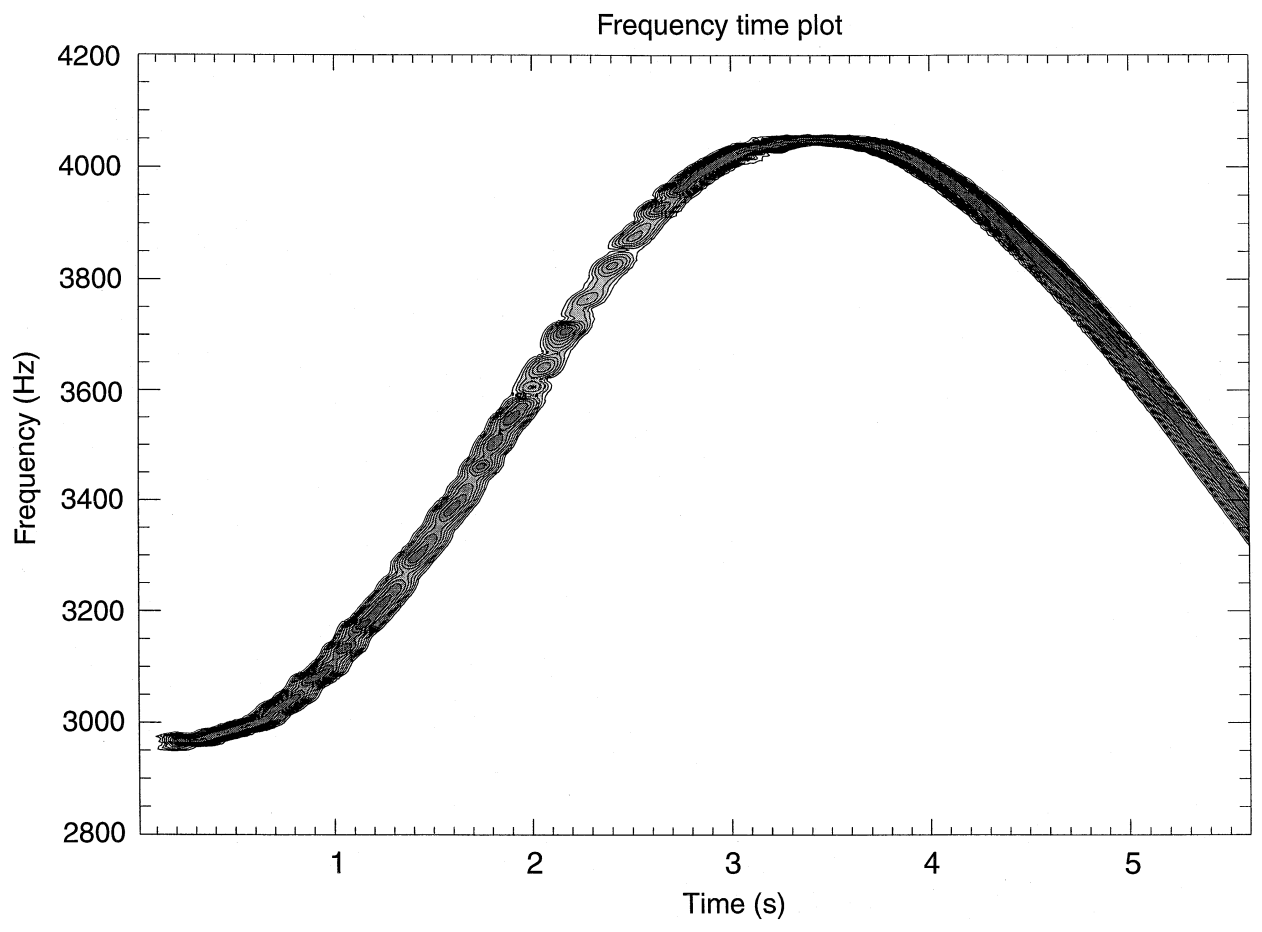

Fig. 14. Case C. Downward hook. Frequency/time diagram of the exit wave field. At $t=3 \mathrm{~s}$ the riser GR transforms itself into a faller GR. At higher frequencies the higher linear growth rates cause the wave profile to creep upstream, which triggers the GR change 

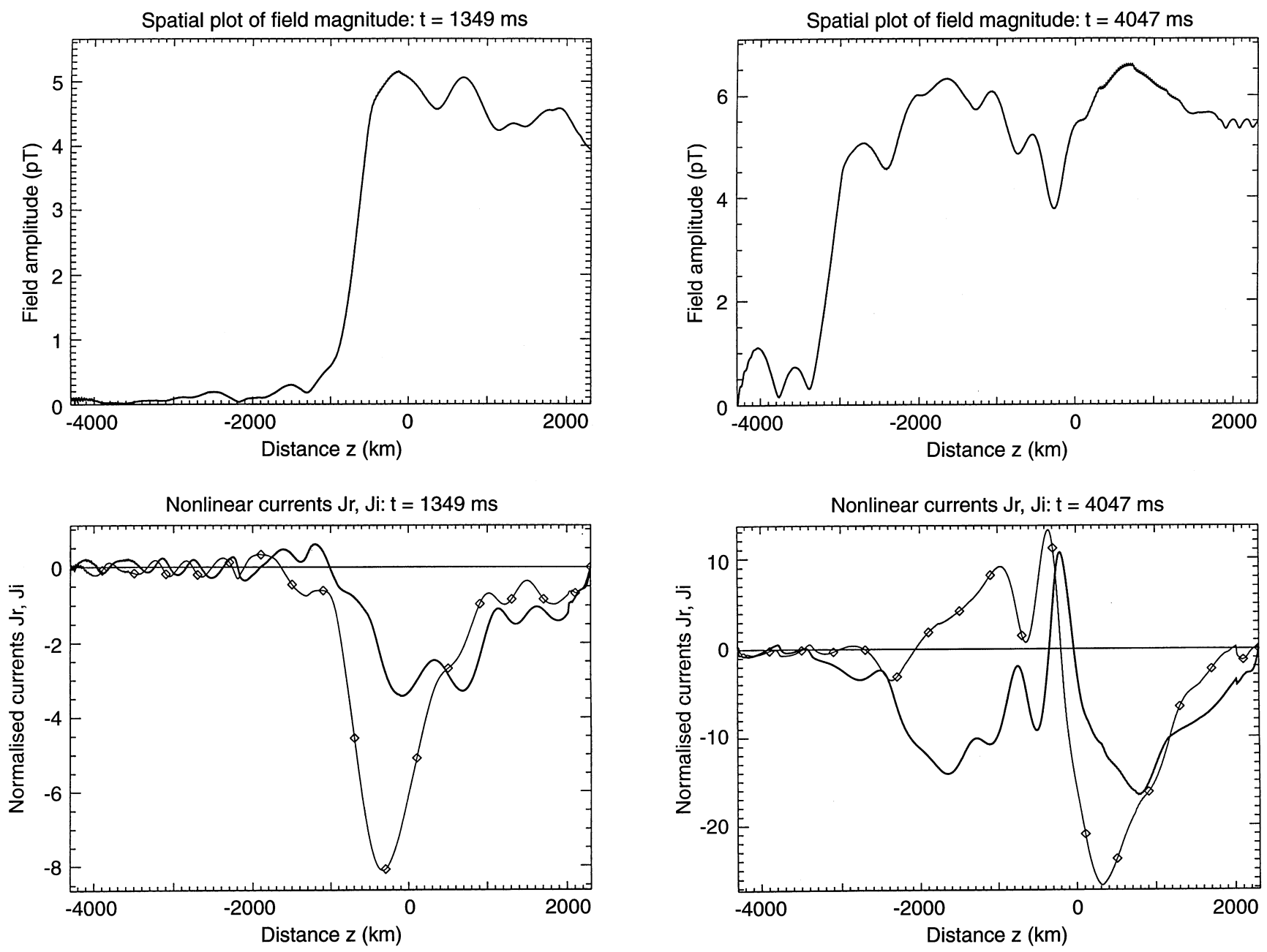

Fig. 15. Case C. Snapshot of riser type GR at $t=1349 \mathrm{~ms}$

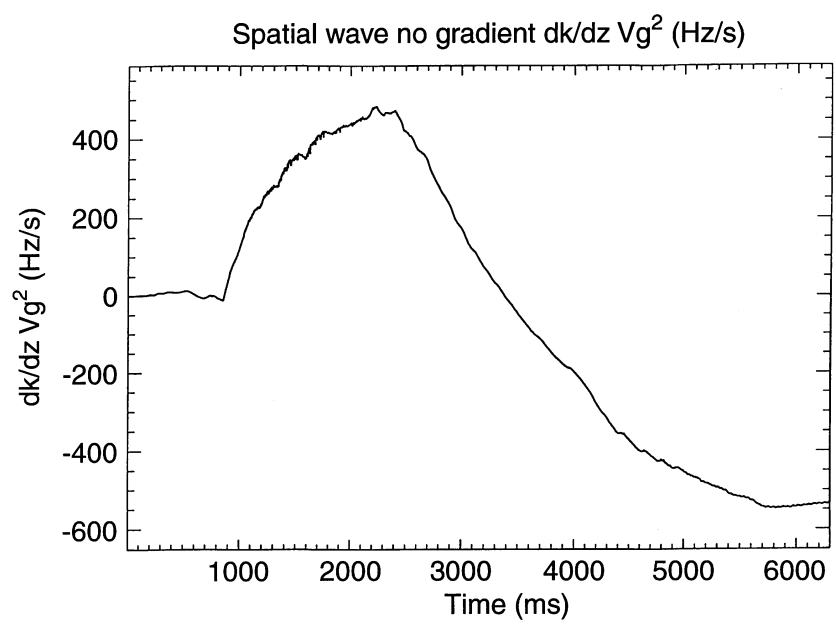

Fig. 17. Case C. Plot of spatial wave number gradient as a function of time. The change from $+400 \mathrm{~Hz} / \mathrm{s}$ to $-400 \mathrm{~Hz} / \mathrm{s}$ at $t=3 \mathrm{~s}$ is entirely expected

inent lines seen at Porojärvi are derived from the Finnish power system, and are doublets, with separation $100 \mathrm{~Hz}$, repeated every $600 \mathrm{~Hz}$. These doublets trigger steep

Fig. 16. Case C. Snapshot of faller type GR at $t=4047 \mathrm{~ms}$

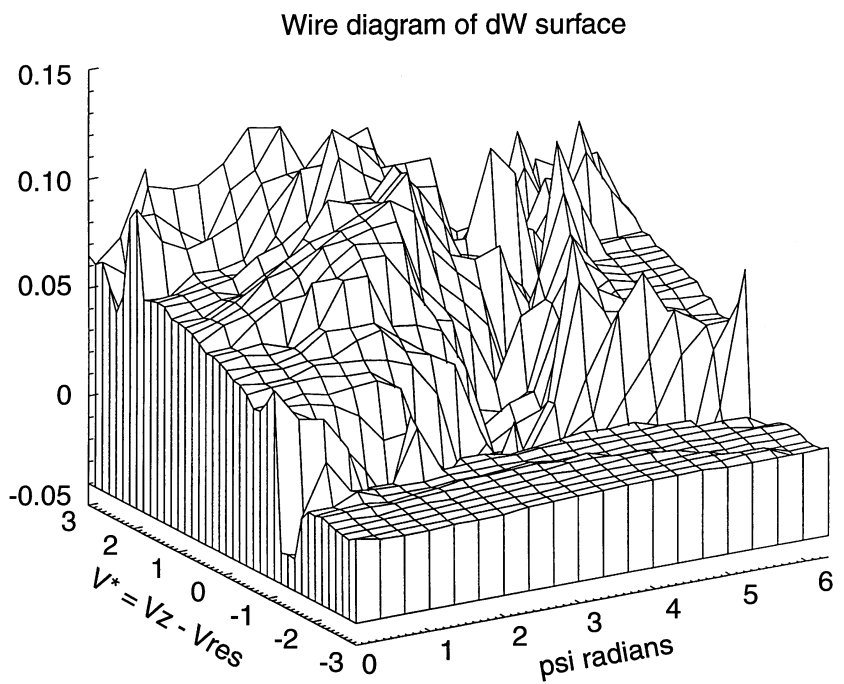

Fig. 18. Case C. Wire diagram of $\Delta W$ at $t=3 \mathrm{~s}$ and at $z=+200 \mathrm{~km}$. Trapped particle topology is more complex in this case

risers preferentially, but we have seen fallers and hooks in the data. Frequency sweep rates of emissions are of order $1 \mathrm{kHz} / \mathrm{s}$, but at higher frequencies very steep risers 


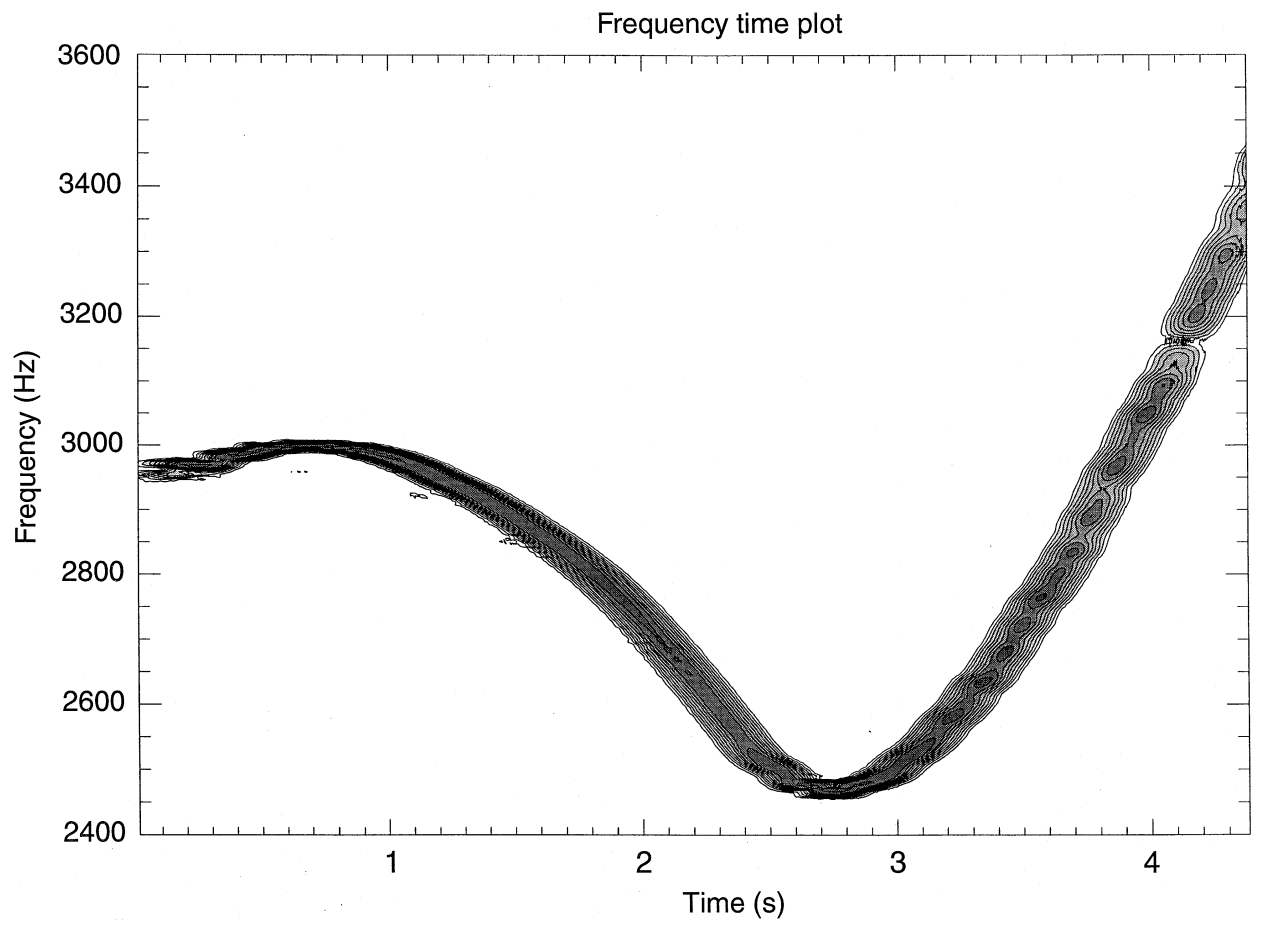

Fig. 19. Case D. Upward hook.

Frequency/time diagram of exit wave field
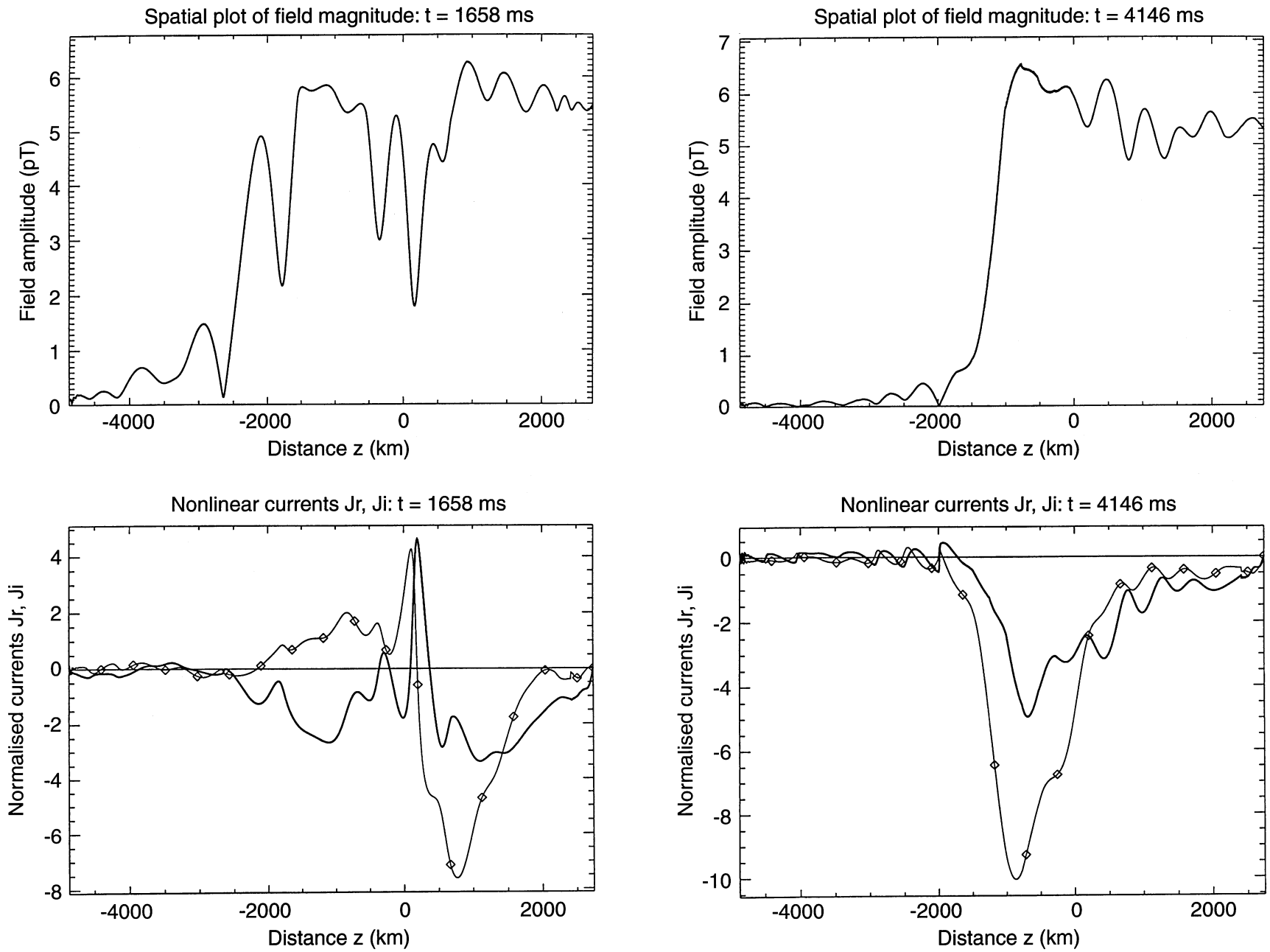

Fig. 20. Case D. Snapshot of GR at $t=1658 \mathrm{~ms}$. This GR is clearly of the faller type

Fig. 21. Case D. Snapshot of the GR at $t=4146 \mathrm{~ms}$. This GR is clearly of the riser type 


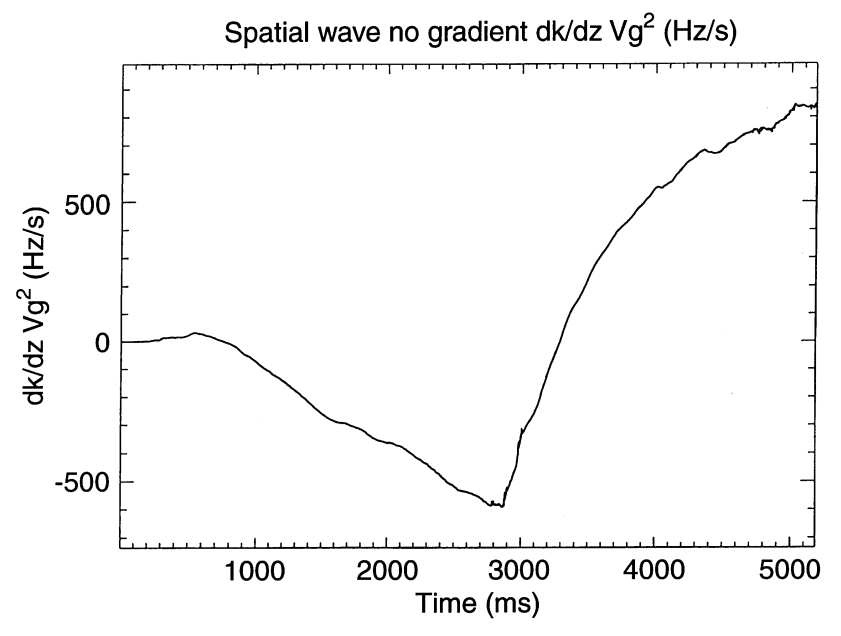

Fig. 22. Case D. Plot of spatial gradient of wave number as a function of time. The value falls to $-500 \mathrm{~Hz} / \mathrm{s}$ in the faller segment and then switches to $+800 \mathrm{~Hz} / \mathrm{s}$ in the subsequent riser segment

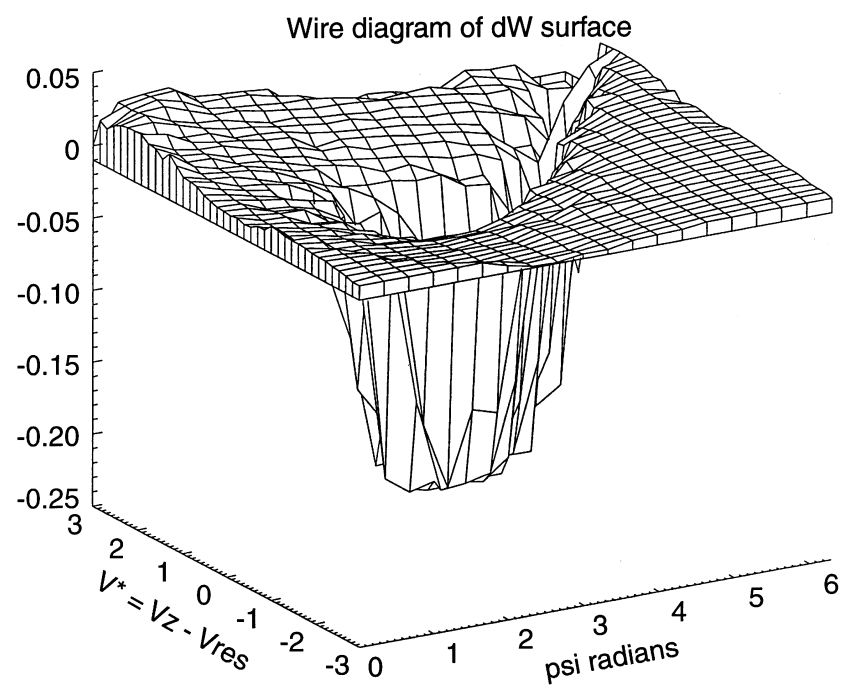

Fig. 23. Case D. Wire diagram of $\Delta W$ in the faller segment at $t=1.5 \mathrm{~s}$, at $z=-1000 \mathrm{~km}$. The distinct bunch of trapped particles with $\Delta W<0$ is again apparent

are common with sweep rates $\sim 2 \mathrm{kHz} / \mathrm{s}$. The injected PLHR waves are moderately weak, and so clearly considerable amplification $(\sim 25 \mathrm{~dB})$ first takes place in the linearly unstable ambient plasma. In the case of the strong doublets, the amplified PLHR lines reach nonlinear amplitudes, at which point nonlinear amplification occurs, giving rise to triggered emissions. PLHR triggered emissions and chorus will result in energetic electron precipitation. Via this mechanism the weak man-made PLHR signals may result in significant transfer of energy from magnetosphere to ionosphere, constituting a significant perturbation of the terrestrial/ near space environment.

Our aim has been to elucidate the nonlinear plasma kinetic processes causing emission triggering by PLHR. We have done this by numerically modelling the process, and aiming to reproduce observed events. The code has been very successful in produced stable long enduring emissions with observed frequency sweep rates. The code shows a preference towards triggering risers as observed. If the linear growth rate is increased to $>100 \mathrm{~dB} / \mathrm{s}$, and the simulation bandwidth narrowed, the code will reliably trigger fallers. The code naturally produces hooks of both types, and is particularly prone to produce upward hooks as input power falls with falling frequency. The basic plasma physical mechanism involved in TEs is the nonlinear cyclotron resonant electron trapping in a parabolic $B$ field inhomogeneity. The fact that the code can reproduce the main types of emission observed with the correct sweep rates, tends to confirm this theoretical assumption.

As for the code itself, it is of necessity only 1D. The simulation method employed, Vlasov Hybrid Simulation, is extremely well suited to this problem, and highly efficient. A number of simulation parameters have to be guessed. The $L$ shell value of the duct in question is not known, but consideration of the frequencies involved suggests that the duct is inside the plasmapause and to the south of Porojärvi. We select varied $L$ values in the range 4-5.5. Similarly equatorial gyrofrequencies are taken between $8.8-13 \mathrm{kHz}$, and electron densities between 250 $400 \mathrm{~cm}^{-3}$. The simulations are not particularly sensitive to these three parameters. Two other parameters are partially determined by plasma physical considerations. The saturation amplitude $B_{\max }$ is taken to be 2-3 times the value at which nonlinearity just starts. The linear growth rate at the equator is taken to be 1.3-2.2 times the level at which the plasma becomes nonlinearly absolutely unstable. The choice of distribution function-multiple biMaxwellian-resembles those actually measured. Fortunately the detailed form of Fo is not critical.

The same VHS code has been used to simulate chorus and emissions at widely different $L$ shells. Nunn and Smith (1996) simulate whistler triggered emissions observed at Halley Bay, and Smith and Nunn (1997) simulate discrete emissions observed at Halley Bay. All these simulations report good agreement with observation. Of greatest interest are the simulations of Geotail chorus at $L=10$. (Nunn et al., 1997). Geotail wave amplitudes are very large and strongly nonlinear, and the code was able to reproduce very closely risers and fallers observed, using all the parameters measured on board. This result provides definite confirmation of the fact that non linear electron cyclotron resonance is indeed the root cause of triggered emissions and chorus. Interestingly, key theoretical aspects of the problem are common to all four cases. These aspects are: (1) the onset of nonlinear absolute instability when the linear growth rate exceeds a certain threshold; (2) The stable nonlinear structure of the GR, and the way that it is the natural end point of the nonlinear growth phase; (3) the distinctive structures of the riser and faller GRs; and (4) spontaneous transformation between GR types and the formation of hooks.

Much work remains to be done on the theoretical aspects of this problem. The real problem is, of course, 3D, and the GR sits within a narrow duct of some kind. The hypothesis of nonlinear unducting loss should be 
verified with a $3 \mathrm{D}$ code, though the computational requirements remain awesome. The possibility of nonlinear unducting loss has been given some support recently. It has been suggested (H Strangeways, personal communication) that unexpectedly large particle precipitation ionospheric footprints could be explained by heavy nonlinear wave leakage from the duct in the equatorial zone. The generation of Langmuir waves and their possible involvement in the overall particle dynamics should be looked at further. This is a soluble problem. As for PLHR itself, the next problem to be tackled should be that of the upward drift of magnetospheric lines. This could be due to repeated nonlinear wpi as the wave field crosses the equatorial zone. This suggestion can be verified by running the 1D VHS code with delayed recirculation of the wave field. Preliminary results have produced a slow upward drift in frequency of magnetospheric lines.

Acknowledgements. The authors wish to gratefully acknowledge the financial support of INTAS contracts 94-2753/. One author, Dr D Nunn, wishes to thank the Radio Atmospheric Science Centre, Kyoto University, for the use of the KDK computer system for code development and for some production runs. The authors thank Mr. J. Kultima, E. Saviaro, and K. Nuutinen for their assistance during the campaign. The authors are also grateful to the Finnish Frontier Guard for arranging the transportation to the measurement site.

Topical Editor K.-H. Glassmeier thanks R. Horne and Y. Omura for their help in evaluating this paper.

\section{References}

Bell, T. F., J. P. Luette, and U. S. Inan, ISEE1 observations of VLF line radiation in the Earth's magnetosphere, J. Geophysical Res., 87, 3530-36, 1982.

Bespalov, P. A., and V. Y. Trakhtengerts, Cyclotron instability of the Earth's radiation belts, Rev. Plasma Phys, 10, 155-212, 1986.

Bullough, K., Satellite observations of power line harmonic radiation, Space Sci. Rev., 35, 175-183, 1983.

Candy, J., A numerical method for solution of the generalised Liouville equation, J. Comput. Phys., 129, 160-169, 1996.

Helliwell, R. A., Whistlers and related ionospheric phenomena, Stanford University Press, Stanford, Calif., USA, 1965.

Helliwell, R. A., Controlled stimulation of VLF emissions from Siple station, Antarctica, Radio Sci, 18, 801-814, 1983.

Helliwell, R. A., J. P. Katsufrakis, T. F. Bell, and R. Raghuram, VLF line radiation in the earth's magnetosphere and its association with power system radiation, J. Geophys. Res, 80 , 31, 4249-58, 1975.

Karpman, V. I., J. N. Istomin, and D. R. Shklyar, Nonlinear theory of a quasi monochromatic whistler mode wave packet in inhomogeneous plasma, Plasma Phys., 16, 685, et seq 1974.

Kikuchi, H., Power line transmission and radiation, Space Sci. Rev., 35, 59-81, 1983.

Klimas, A. J. and W. M. Farrell, A splitting algorithm for Vlasov simulation with filamentation filtration, J. Computat. Phys., 110, 150-163, 1994.

Luette, J. P., C. G. Park, and R. A. Helliwell, The control of magnetospheric chorus by power line radiation, J. Geophys. Res., 84, 2657-2660, 1979.

Matthews, J. P., and K. Yearby, Magnetospheric line radiation observed at Halley, Antarctica, Planet. Space Sci., 29, 97-105, 1981.

Molchanov, O. A., M. Parrot, M. M. Mogilevsky, and F. LeFeuvre, A theory of PLHR emissions to explain the weekly variation of
ELF data observed by a low-altitude satellite, Annales Geophysicae, 9, 669-680, 1991.

Nunn, D., A nonlinear theory of sideband stability in ducted whistler mode wave, Plane. Space Sci., 34, 5, 429-451, 1986.

Nunn, D., The numerical simulation of VLF non-linear wave particle interactions in collision free plasmas using the Vlasov hybrid simulation technique, Comput Phys Comm, 60, 1-25, 1990.

Nunn, D., A novel technique for the numerical simulation of hot collision free plasma; Vlasov hybrid simulation, J. Computat. Phys., 108, 1, 180-196, 1993.

Nunn, D., Y. Omura, H. Matsumoto, I. Nagano, and S. Yagitani, The numerical simulation of VLF chorus and discrete emissions observed on the Geotail satellite using a Vlasov code, $J$. Geophys. Res., 102, 27083-27097, 1997.

Nunn, D., Smith A. J., Numerical simulation of VLF risers, fallers and hooks observed in Antarctrea, J. Geophys. Res., 103, A4, 6771-6784, 1998.

Omura. Y., and H. Matsumoto, Computer simulations of basic processes of coherent whistler wave particle interactions in the magnetosphere, J. Geophys. Res., 87, 4435, et seq. 1982.

Omura, Y., H. Matsumoto, T. Miyake, and H. Kojima., Electron beam instabilities as generation mechanism of electrostatic solitary waves in the magnetotail J. Geophys. Res., 101, 26852697, 1996.

Park, C. G., and D. C. D. Chang, Transmitter simulation of power line harmonic radiation effects in the magnetosphere, Geophys. Res. Lett., 5, 861-864, 1978.

Park C. G., and R. A. Helliwell, Power line radiation in the magnetosphere, Adv. Space Res., 1, 423-437, 1981.

Park, C. G., R. A. Helliwell, and F. LeFeuvre, Ground observations of power line radiation coupled to the ionosphere and magnetosphere, Space Sci. Rev., 35, 131-137, 1983.

Parrot, M., and Y. Zaslavski, Physical mechanisms of man-made influences on the magnetosphere, Surv. Geophy., 17, 67-100, 1996.

Rodger, C. R., N. R. Thomson, and R. L. Dowden., VLF line radiation observed by satellite, J. Geophys. Res., 100, 5681$5689,1995$.

Shklyar, D. R., D. Nunn, A. J. Smith, and S. S. Sazhin, An investigation into the nonlinear frequency shift in magnetospherically propagated pulses, J. Geophys. Res., 97, 19 38919 402, 1992.

Smith, A. J., and D. Nunn, Numerical simulation of VLF risers, fallers and hooks observed in Antarctica, J. Geophys. Res., 103, 6771-6784, 1998.

Thorne, R. M., and B. T. Tsurutani, Power line harmonic radiation: can it significantly affect the Earth's radiation belts?, Science, 204, 839-841, 1979.

Thorne, R. M., and B. T. Tsurutani, Comment on 'Sunday decreases in magnetospheric wave activity' by C. G. Park and T. R. Miller, J. Geophys. Res., 86, 1639-1641, 1981.

Trakhtengerts, V. Y., Magnetosphere cyclotron maser:BWO generator regime, J. Geophys. Res., 100, 17205-210, 1995.

Trakhtengerts V. Y., M. J. Rycroft, and A. G. Demekho., Interrelation of noiselike and discrete ELF/VLF emissions generated by cyclotron interaction, J. Geophys. Res., 101, 13 293-13 301, 1996.

Tsurutani, B. T. and R. M. Thorne, A skeptic's view of PLR effects in the magnetosphere, Adv. Space Res., 1, 439-444, 1981.

Tsurutani, B. T., S. R. Church, and R. M. Thorne, A search for geographic control on the occurrence of magnetospheric ELF emissions, J. Geophys. Res, 84, 4116-4124, 1979.

Yearby, K. H., J. P. Matthews, and A. J. Smith, VLF line radiation observed at Halley and Siple, Adv. Space Res., 1, 445-448, 1981.

Yearby, K. H., and A. J. Smith, The polarisation of whistlers received on the ground near $L=4$. J, Atmos. Terre. Phys., 56 (11), 1499-1512, 1994.

Yearby, K. H., A. J. Smith, T. R. Kaiser, and K. Bullough, Power line harmonic radiation in Newfoundland, J. Atoms. Terr. Phys., 45, 409-419, 1983. 